\title{
Sostenibilidad económica y situación financiera de las estaciones de esquí alpino del Pirineo catalán
}

\author{
Laura Sánchez Pulido(iD, Natalia Daries Ramón (iD, Eduard Cristóbal Fransi (ii \\ Universidad de Lleida (Spain) \\ lsanchez@aegern.udl.cat,ndaries@aegern.udl.cat,_ecristobal@aegern.udl.cat
}

Received June, 2016

Accepted October, 2016

\section{Resumen}

Objeto: El presente artículo tiene como objetivo analizar la rentabilidad y la situación financiera de las principales estaciones de esquí alpino catalanas, con el fin de comprobar si éstas son sostenibles desde un punto de vista económico, teniendo en cuenta los factores externos que le afectan.

Diseño/metodología/enfoque: Con el fin de realizar el estudio de rentabilidad y situación financiera, se obtuvieron los estados financieros de las principales estaciones de esquí Alpino del Pirineo Catalán: Baqueira-Beret, Masella, Boí Taüll, Vallter 2000, La Molina, Vall de Núria y Espot Esquí-Port Ainé, para los períodos comprendidos entre 2011-2014 y se procedió a aplicar una serie de ratios económicos y de estructura con el fin de establecer un diagnóstico sobre su situación.

Aportaciones y resultados: Los resultados ponen de manifiesto que las estaciones de Baqueira-Beret y Masella son las únicas que obtienen beneficios, mientras que el resto de estaciones obtienen pérdidas en todos los períodos analizados. Respecto a la situación financiera, los resultados revelan que el nivel de endeudamiento de las estaciones es, en general reducido, llegando a la conclusión de que la mayoría de ellas no son sostenibles desde un punto de vista estrictamente económico. 
Limitaciones: Las principales limitaciones del trabajo son por un lado que las conclusiones se obtienen a partir de un período de análisis de cinco años (2011-2015), y en segundo lugar, se circunscriben a una región determinada.

Implicaciones prácticas: Los resultados de este trabajo pueden ayudar a tomar decisiones tanto a los gestores de las estaciones de esquí como a los administradores públicos para implementar estrategias para conseguir diversificar la oferta y mejorar los resultados.

Implicaciones sociales: En la actualidad la mayoría de las estaciones han sido rescatadas o adquiridas por la Administración, por lo que se deduce que se mantienen por las rentas que genera su existencia. Es decir, por las externalidades positivas que genera en el territorio donde se ubican.

Originalidad / Valor añadido: La presente investigación se centra en el estudio financiero de un sector poco estudiado como son las estaciones de esquí y el turismo de nieve con la enorme importancia que tiene en la economía de la zona y en el desarrollo territorial. Los agentes interesados son tanto las administraciones públicas como los gestores de este tipo de empresas.

Palabras clave: Turismo, Nieve, Sostenibilidad económica, Esquí, Rentabilidad

Códigos JEL: G00, G10, Z33

Title: Economic sustainability and financial situation of the alpine ski resorts on the Catalan Pyrenees

\section{Abstract}

Purpose: The present work seeks analyze the profitability and the financial situation of the main Catalan alpine ski resorts, with the objective to check if these are sustainable from an economic point of view, taking into account the external factors that affect him.

Design/methodology: With the objective to carry out the study of profitability and financial situation, we obtained the financial states of the main Alpine ski resorts of the Catalan Pyrenees: Baqueira-Beret, Masella, Boí Taüll, Vallter 2000, La Molina, Vall de Núria and Espot Ski-Port Ainé, for the periods comprised between 2011-2015 and we proceeded to apply a series of economical and structural ratios with the purpose of establish a diagnostic on his situation. 
Findings: The results show that the Baqueira-Beret and Masella resorts are the only that obtain profits, whereas the rest of resorts obtain losses in all the periods analyzed. With regard to the financial situation, the results reveal that the level of indebtedness of the resorts is, in general reduced, arriving to the conclusion that the majority of them are not sustainable from a strictly economic point of view.

Research limitations/implications: The main limitations of this work are on the one hand that the conclusions are obtained from a period of analysis of five years (2011-2015), and the second, they are circumscribed to a determinate region.

Practical implications: The results of this work can help to take decisions so much to the ski resorts managers as to the public administrators for implementing strategies to achieve diversify the offer and improve the results.

Social implications: Nowadays the majority of the ski resorts have been rescued or purchased by the Administration. We can deduce that it keeps by the incomes that generate his existence. That is to say, by the positive externalities that generates in the territory where they are situate.

Originality/value: The present research focuses in the financial study of a little studied sector as they are the ski resorts and the snow tourism with the enormous importance that has in the economy of the region and in the territorial development. The agents interested are so much the public administrations like the managers of this type of firms.

Keywords: Tourism, Snow, Economic sustainability, Ski, Profitability

Jel Codes: G00, G10, Z33

\section{Introducción}

El turismo de nieve lo podemos clasificar dentro del turismo deportivo y del turismo de naturaleza, ya que esta disciplina se genera motivada por el deseo de practicar actividades deportivas en un entorno natural, de nieve y montaña (Esteban-Talaya, 1995). Por otro lado Saz y Carús (2008), acuñan el concepto de Segmento Turístico Recreativo de Alta Montaña (STRAM) y lo definen como "el formado por Centros Turísticos Recreativos de Alta Montaña (CTRAM) o destinos que a través de una inversión 
previa en recursos humanos, equipos e infraestructura diversa explotan en ubicaciones de alta montaña, también fuera de la época invernal, centros recreativos o de ocio".

A nivel territorial, las actividades turísticas, especialmente las relacionadas con el esquí han sido ampliamente promovidas des de los años 50 en las zonas montañosas españolas, como los Pirineos, para el desarrollo económico de las mismas (Lasanta, Laguna \& Vicente-Serrano, 2007). La oferta española de turismo de nieve y de montaña representa un total de 1.039 kilómetros de pistas esquiables, una cifra que llama la atención al compararse con los $3.168 \mathrm{~km}$ de costa española. Para contextualizar el sector, es interesante visionar el mapa topográfico de la península ibérica elaborado por ATUDEM, en el que se aprecia la distribución espacial de las diferentes estaciones, en todas las cordilleras de la península con elevación considerable sobre el nivel del mar. Así vemos que las estaciones de esquí son una realidad que afecta a un gran número de CC.AA. (Andalucía, Aragón, Asturias, Cantabria, CastillaLeón, Cataluña, Madrid, Galicia, La Rioja y Navarra).

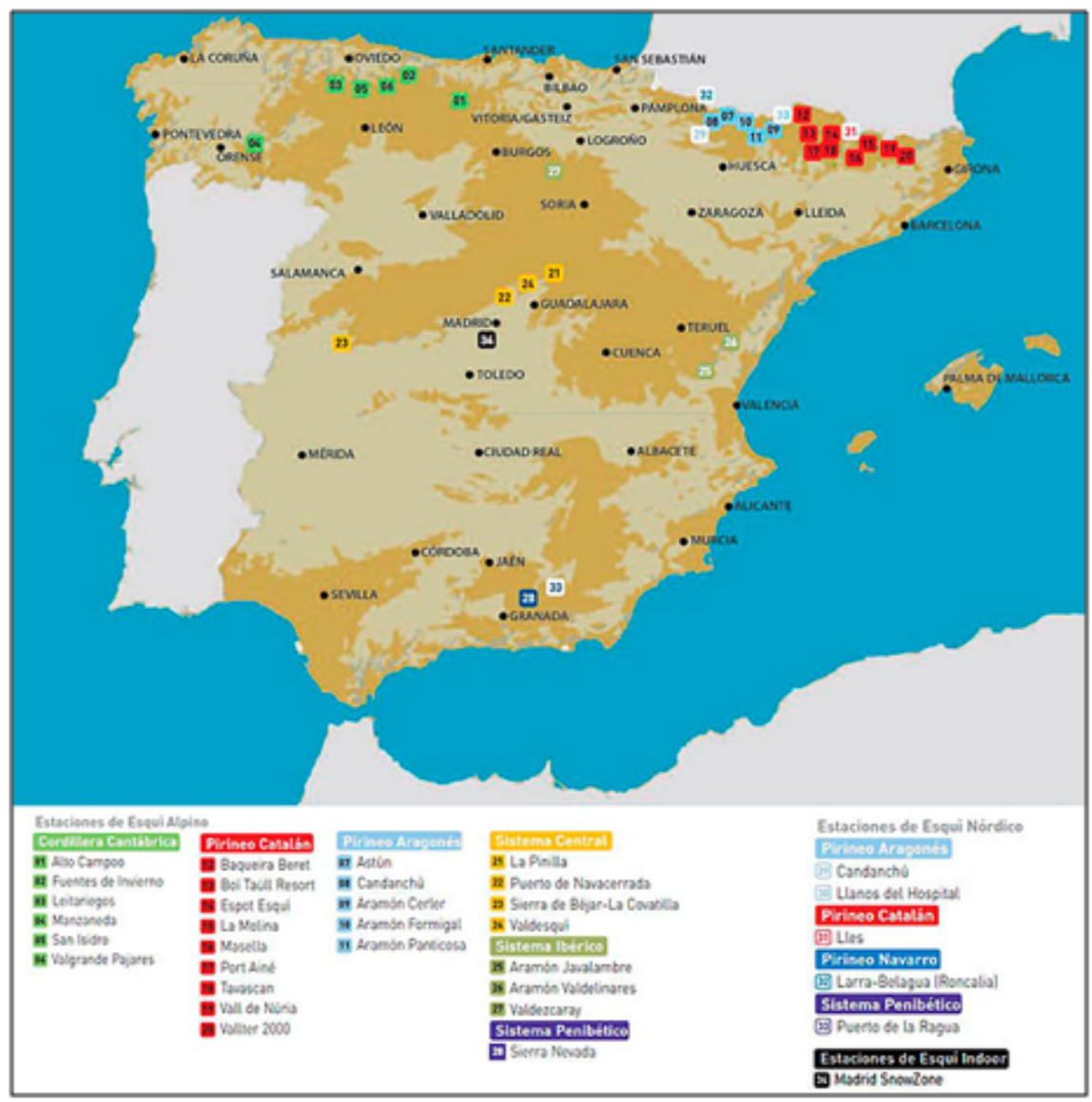

Figura 1. Mapa de las Estaciones de esquí en España (ATUDEM, 2016). Mapa de ubicación de las estaciones de esquí españolas. Documento disponible en:

http://www.atudem.es/mapa ubicacion.aspx 
La mayoría de las estaciones de esquí se circunscriben en la modalidad de estaciones de esquí alpino, pero un gran número de las estaciones enumeradas en este mapa, son a su vez estaciones de esquí nórdico. El esquí alpino es una modalidad más conocida y moderna que consiste en descender por una montaña nevada mientras que el esquí nórdico consiste en desplazarse de un sitio a otro. En el sector de las estaciones de esquí, las de esquí nórdico suponen un porcentaje significativamente inferior tanto a nivel numérico como económico. Por ello en el estudio que desarrollamos nos centraremos en las estaciones de esquí alpino.

En conjunto, las estaciones de esquí españolas representan un sector económico que, en los últimos diez años, ha destinado más de 700 millones de euros a la inversión en mejoras de sus estaciones y cuenta con un creciente número de deportistas y turistas que visitan las estaciones o que realizan jornadas de esquí. Este sector desarrolla a su vez actividades económicas colaterales como transporte, hostelería, comercio de ropa y complementos, alquiler y venta de material deportivo, etc. (García-Abad, 2012).

Y todo esto a pesar de la crisis económica que ha sufrido nuestro país en los últimos años, factor externo que ha afectado a todos los sectores en general y ha influido en los resultados empresariales de las estaciones de esquí. Tal como se puede observar en la Figura 2, en 2007 las estaciones que más notan los efectos de la crisis en su nivel de ventas son Baqueira-Beret, Masella y Vallter 2000. En cambio, si ampliamos el horizonte temporal, es la estación de Boí Taüll la que sufre un descenso más pronunciado en sus resultados.

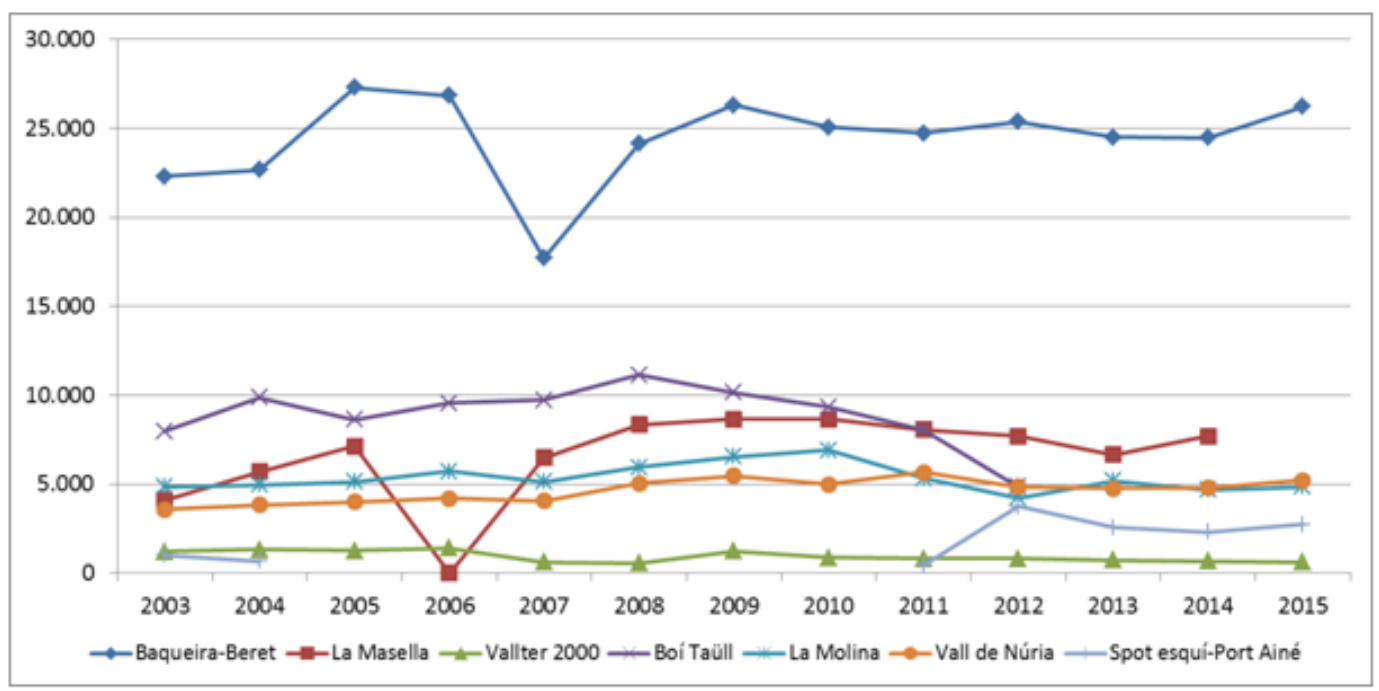

Figura 2. Importe neto de la cifra de negocios de las estaciones de esquí analizadas (2003-2015). (SABI y Memorias anuales de FGC) 
Por otro lado y si nos centramos en los resultados de negocio, las únicas estaciones que obtienen beneficios durante todo el período 2003-2015, son las de Baqueira-Beret y Masella. Si bien es cierto que, en los años 2011 y 2012 Baqueira-Beret sufre una disminución en sus beneficios. En cambio, Masella obtiene peores resultados en los ejercicios 2013 y 2014, aun así mantiene un resultado de ejercicio con signo positivo. El resto de estaciones, como se observa en la Figura 3, obtienen pérdidas en prácticamente la totalidad de los años analizados. Lo que pone de manifiesto que son deficitarias tanto en los años de crisis económica como en los predecesores. Se observa que, excepcionalmente, la estación de Boí Taüll en 2004 registra un resultado del ejercicio positivo (beneficios) procedentes de resultados extraordinarios.

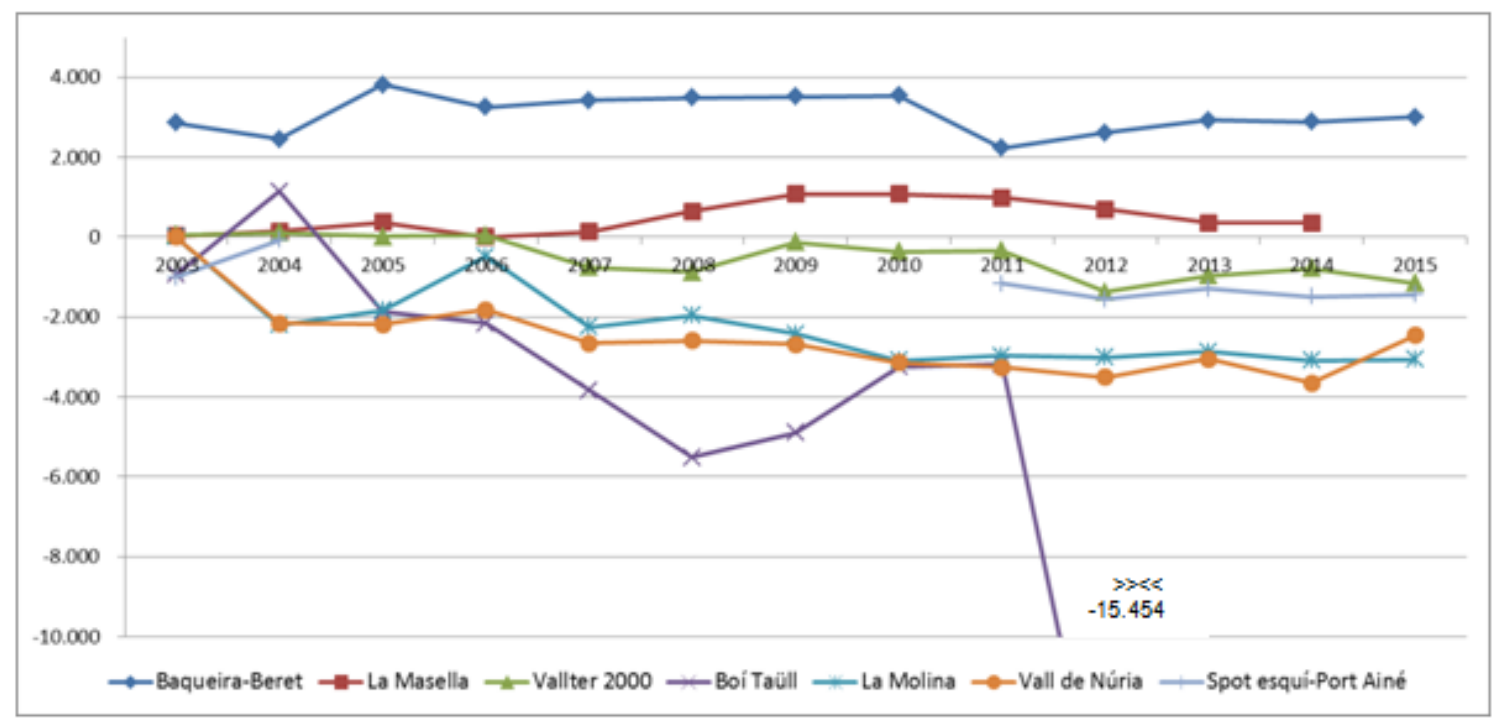

Figura 3. Resultados de negocio de las estaciones de esquí analizadas (2003-2015).

(SABI y Memorias anuales de FGC)

Según datos de ATUDEM (2016), el número de visitantes a las estaciones de esquí, superan los cinco millones de personas y ofrecen una oferta de alojamiento de más de 217,000 plazas. Por tanto, los datos que documentan el flujo de turistas hacia las zonas de montaña ponen de manifiesto la importancia económica del sector (Saz \& Carús, 2008). 


\begin{tabular}{|c|c|c|c|c|c|c|c|c|c|}
\hline Temporada & 2004-05 & 2005-06 & $2006-07$ & 2007-08 & 2008-09 & 2009-10 & 2010-11 & 2011-12 & $20-2013$ \\
\hline $\begin{array}{l}\text { Número de } \\
\text { visitantes }\end{array}$ & $6,254,715$ & $7,165,109$ & $4,559,144$ & $5,160,195$ & $6,657,778$ & $5,618,059$ & $5,541,159$ & $4,505,715$ & $5,110,000$ \\
\hline $\begin{array}{l}\text { Capacidad total } \\
\text { de transporte } \\
\text { (persona/hora) }\end{array}$ & 384,858 & 392,182 & 421,660 & 459,747 & 467,677 & 456,748 & 454,852 & 449,457 & 460,300 \\
\hline $\begin{array}{l}\text { Pistas Balizadas } \\
(\mathrm{km})\end{array}$ & 887 & 888 & 953 & 332 & 1,036 & $1,007,42$ & $1,011,96$ & $1,026,82$ & $1,039,50$ \\
\hline $\begin{array}{l}\text { Producción } \\
\text { nieve artificial } \\
(\mathrm{km})\end{array}$ & 274 & 292 & 321 & 333 & 358 & 339 & 349 & 365 & 375 \\
\hline $\begin{array}{l}\text { Oferta } \\
\text { alojamiento (1) }\end{array}$ & 133,153 & 163,996 & 174,138 & 176,482 & 220,026 & 216,452 & 213,699 & 240,911 & 217,400 \\
\hline $\begin{array}{l}\text { Inversiones } \\
\text { (miles de euros) }\end{array}$ & 11,845 & 73,056 & $99,510,291$ & $72,596,173$ & $50,365,331$ & $54,291,895$ & $59,724,303$ & $24,372,277$ & - \\
\hline
\end{tabular}

Tabla 1. Cifras en las Estaciones de Esquí Alpino. (1) Hoteles, apartamentos, albergues y casas rurales, incluida área de influencia $($ radio $=35 \mathrm{Km}$.). (ATUDEM, 2016)

Siguiendo el trabajo de Falk (2010), la demanda de turismo de invierno depende de diversos factores: la renta nacional e internacional, los precios, el transporte, los costes, la ubicación de las vacaciones de Pascua y el cambio climático. El cambio climático se revela como un factor importante, sobre todo para algunas estaciones de esquí de baja altura. En estas estaciones de esquí, el descenso de la demanda es mucho más pronunciada, ya que son muy dependientes del grosor de la nieve (Falk, 2010). De esta manera, las empresas invierten sobre todo en estaciones de esquí de altura con el objetivo de crear grandes dominios esquiables (Gerbaux \& Marcelpoil, 2006; Falk, 2009) para atraer a esquiadores extranjeros (franceses, ingleses y rusos).

Una vez vista la importancia significativa del turismo de nieve consideramos necesario evaluar su sostenibilidad económica. Por ello, el principal objetivo del presente trabajo es analizar a través de un análisis económico financiero la viabilidad de las principales estaciones de esquí Alpino teniendo presente los factores externos que le afectan y que el alcance del estudio se limita al Pirineo catalán. 


\section{Impacto socio-económico, ambiental y demográfico de las estaciones de esquí}

En el caso del Pirineo, el impacto socio-económico de las estaciones de esquí ha sido desigual, a la vez que ha conllevado unos impactos ambientales asociados al abandono del sector primario y una transición hacia una economía fuertemente dependiente del turismo de esquí principalmente en las proximidades de las estaciones (Lasanta et al., 2007). Desde mediados del siglo XX se potenció el turismo de nieve en el Pirineo Catalán, dada la profunda crisis sufrida en la agricultura y la ganadería y la migración de la población de las zonas rurales a las ciudades, la estrategia que se llevó a cabo fue considerar las estaciones de esquí como principal motor de desarrollo.

Transcurrido medio siglo hay trabajos como el realizado por Lasanta (2010) en el Pirineo Aragonés que han analizado el efecto de la implantación de las estaciones de montaña y llegan a la conclusión que los resultados son positivos respecto a la situación demográfica y negativos en la evolución del sector primario. Los municipios con estaciones de esquí incrementan su población, sin embargo disminuyen sus activos referentes al sector primario, creando cierta dependencia del sector servicios.

Siguiendo la línea del trabajo de Lasanta (2010), y tal como se observa en la Tabla 2, si bien la población de las comarcas de montaña con estaciones de esquí ha aumentado desde la década pasada, es cierto que durante la crisis ha habido un cierto decrecimiento aunque en menor medida que en el conjunto de la zona pirenaica.

\begin{tabular}{|c|c|c|c|c|c|c|c|c|c|c|}
\hline Comarcas & Estaciones & 2002 & 2003 & 2004 & 2007 & 2008 & 2009 & 2013 & 2014 & 2015 \\
\hline Alta Ribagorza & BoíTaüll & 3,655 & 3,687 & 3,796 & 4,123 & 4,332 & 4,375 & 4,097 & 3,873 & 3,884 \\
\hline Cerdaña & Masella/Molina & 15,266 & 15,540 & 16,065 & 17,744 & 18,658 & 18,656 & 18,630 & 18,063 & 17,870 \\
\hline PallarsSobirà & Port Ainé/Spot & 6,301 & 6,396 & 6,666 & 7,191 & 7,446 & 7,625 & 7,330 & 7,220 & 7,060 \\
\hline Ripollés & $\begin{array}{l}\text { Vallter2000/ } \\
\text { Vall de Núria }\end{array}$ & 26,268 & 26,138 & 26,162 & 25,576 & 26,831 & 26,821 & 25,995 & 25,700 & 25,342 \\
\hline Valle de Arán & BaqueiraBeret & 8,087 & 8,373 & 8,832 & 9,815 & 10,194 & 10,295 & 10,090 & 9,993 & 9,926 \\
\hline $\begin{array}{l}\text { Alto Pirineo y } \\
\text { Arán }\end{array}$ & & 65,537 & 66,665 & 68,386 & 73,906 & 76,287 & 76,828 & 74,882 & 73,557 & 73,044 \\
\hline
\end{tabular}

Tabla 2. Evolución de la población en las comarcas de montaña con estaciones de esquí.

(Instituto Estadístico de Cataluña, 2016)

Por otro lado y en cuanto a la actividad económica, podemos observar la disminución de activos en el sector primario tomando como referencia la evolución del número de explotaciones ganaderas situadas en las comarcas catalanas de montaña. Tal como se indica en la Tabla 3, hay una disminución generalizada respecto a este tipo de explotaciones, exceptuando las del Valle de Arán que presentan un 
claro repunte. En cambio vemos un incremento generalizado de establecimientos hoteleros en este mismo periodo de tiempo, como ejemplo del peso del sector servicios derivado del turismo.

\begin{tabular}{|l|c|r|r|r|r|}
\cline { 3 - 6 } \multicolumn{2}{c|}{} & \multicolumn{2}{c|}{ Explotaciones ganaderas } & \multicolumn{2}{c|}{$\begin{array}{c}\text { Establecimientos } \\
\text { hoteleros }\end{array}$} \\
\hline Comarcas & Estaciones & $\mathbf{1 9 9 9}$ & $\mathbf{2 0 0 9}$ & $\mathbf{1 9 9 9}$ & $\mathbf{2 0 0 9}$ \\
\hline Alta Ribagorza & BoíTaüll & 92 & 91 & 34 & 48 \\
\hline Cerdaña & Masella/Molina & 325 & 291 & 53 & 66 \\
\hline PallarsSobirà & Port Ainé/Spot & 311 & 287 & 59 & 62 \\
\hline Ripollés & Vallter 2000/ Vall de Núria & 489 & 415 & 60 & 61 \\
\hline Valle de Arán & BaqueiraBeret & 148 & 172 & 84 & 105 \\
\hline
\end{tabular}

Tabla 3. Evolución de las explotaciones ganaderas frente a los establecimientos hoteleros en las comarcas catalanas de montaña con estaciones de esquí. (Instituto Estadístico de Cataluña, 2016)

En el Pirineo catalán donde se concentra el turismo de nieve son regiones con menores niveles per cápita y altas tasas de desempleo derivadas de la estacionalidad (Arnáez, Lasanta \& Pascual-Bellido, 2014). Por otro lado, en los últimos años multitud de estudios han evidenciado y corroborado la existencia de un cambio climático global (IPCC, 2014) junto con la eficiencia económica que es otro de los factores genéricos a considerar: en este aspecto, García-Abad (2012) y Massons (2007) destacan que el cambio climático está erosionando de manera alarmante la eficiencia del turismo de nieve.

Siguiendo esta línea, Clarimont (2008) argumenta que frente a las predicciones de aumento de las temperaturas, la producción de nieve artificial se hará con el tiempo menos eficiente y más costoso. Se plantea pues la cuestión de su rentabilidad económica a largo plazo ya que ésta parece incierta. Para las áreas de esquí situada en cotas bajas, la instalación de cañones procede de una estrategia a corto o medio plazo, insostenible económicamente y cuestionable ecológicamente.

No obstante, los efectos del cambio climático serán analizados en mayor profundidad en el siguiente apartado. Retomando los factores económicos genéricos, hay que destacar que el nivel de vida de las comarcas catalanas en donde se concentra el grueso del turismo de nieve, destaca por la desigualdad, en la medida en que existen muchas familias cuyos ingresos dependen en exclusiva del turismo de nieve. 


\section{El efecto del cambio climático en el turismo de nieve}

Las zonas de montaña han sido identificadas como regiones especialmente vulnerables a los efectos del cambio climático (Beniston, 2003). Por un lado, debido al ritmo al que se están produciendo estos cambios, por ejemplo en el incremento de la temperatura media terrestre, superior a la media global (El Kenawy, López-Moreno \& Vicente-Serrano, 2012). Por otro lado, el gradiente altitudinal propio de los territorios de montaña induce a una gran variedad fisiológica y biológica con abruptos cambios en la fauna y la vegetación y con una gran sensibilidad a pequeñas variaciones climáticas (Keller, Kienast \& Beniston, 2000).

La mayoría de autores que han dedicado sus esfuerzos al estudio del turismo de nieve (Gómez-Martín, 2003; Jiménez \& Prats, 2006; López-Palomeque, 1996, 2004; Martín, 2008; Martín, López Palomeque, \& Vide, 2002; Moreno, 2005; Romagosa, Priestley \& Llurdés, 2011; Clarimont, 2008) coinciden en señalar que el impacto del cambio climático ha sido más severo en el turismo de nieve que en el tradicional turismo de Sol y playa.

Moreno (2005), afirma que en el turismo de invierno (turismo de nieve-estaciones de esquî), las consecuencias del cambio climático son, hoy por hoy, más evidentes que en el caso del turismo de sol y playa, y también es más patente su dificultad de adaptación. Se coincide, pues, que en general las zonas de montaña afectadas se muestran más vulnerables que las zonas costeras y las islas.

A pesar de que el cambio climático es sólo uno de los factores que influyen en el turismo de invierno, la presencia o ausencia de nieve, en concreto el espesor de nieve y su temporalidad son elementos claves a la hora de valorar la viabilidad y la sostenibilidad a medio y largo plazo del sector (Pons et al., 2014).

Así, una de las mayores preocupaciones para las empresas que se dedican al turismo de nieve en Cataluña son los cada vez más altos requerimientos de recursos hídricos derivados de la disminución de las nevadas (Clarimont, 2008). En este aspecto, el estudio preliminar de los efectos del cambio climático en España realizado por Moreno (2005) recoge la alarmante tendencia contradictoria que representa el aumento de la demanda hídrica y el descenso de los recursos hídricos. Más aún, los productos turísticos tienen como input este preciado recurso natural (Moreno, 2005).

Recordemos que el acortamiento de la temporada de nieve y la escasez de nieve en las bajas altitudes de las montañas del Pirineo catalán obligan el uso de la innivación artificial, lo cual significa un incremento considerable en los costes de producción y el uso de recursos hídricos. No es de extrañar pues, que la 
WTO (World Tourism Organitation) afirmase que "el impacto del cambio climático sobre la industria del turismo deportivo de nieve es potencialmente grave" (WTO, 2008).

La Figura 4 nos indica el número de días de apertura de las estaciones de esquí analizadas. Estas cifras muestran una gran variación de un año a otro fruto de la diversidad del clima y refleja por tanto, el esfuerzo de las estaciones para alargar al máximo la temporada.

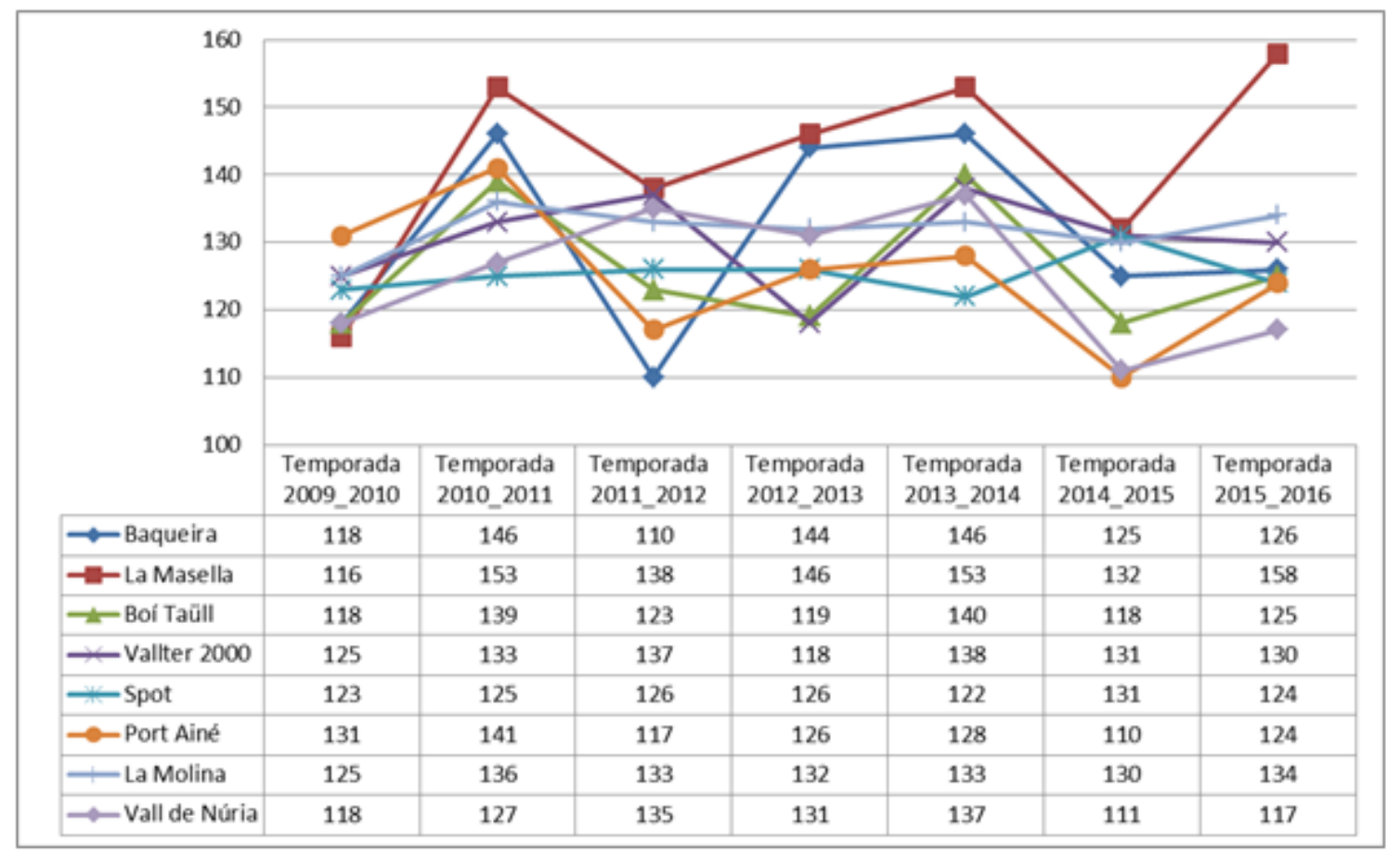

Figura 4. Días de apertura de las estaciones de esquí analizadas (2009-2015). (infonieve.es)

Todos los informes coinciden en señalar que la temperatura media de España ha crecido en torno a 0.9 $\mathrm{C}^{\circ}$ a los largo del siglo XX. Mientras que las previsiones para el siglo XXI son aún más alarmantes. Según un estudio del GIEC (Grupo de Expertos Intergubernamentales sobre la Evolución del Clima) realizado en 2007 cuantificó que las emisiones de gas de efecto invernadero elevarán la temperatura entre $1.8 \mathrm{C}^{\circ}$ y $4 \mathrm{C}^{\circ}$ hasta 2100 . Además, se prevén notables transformaciones en el clima del Pirineo catalán que afectarán, con seguridad, la pluviometría y la innivación de la región (Clarimont, 2008). 
Asimismo, hay que destacar que una de las causas que explican el cambio climático de la Península Ibérica son las emisiones de CO2; si observamos la Figura 5, las emisiones de CO2 per cápita en España exteriorizan una tendencia creciente a largo del periodo que comprende los años 1960 y 2010.

No obstante, se observan descensos en este indicador que derivan de las fases del ciclo económico (Schumpeter, 1927); esto es, la fases de declinación y recesión del ciclo vienen acompañadas de descensos en la emisión de CO2 per cápita, por cuanto derivan de caída de la producción, el nivel de consumo y la inversión privada.

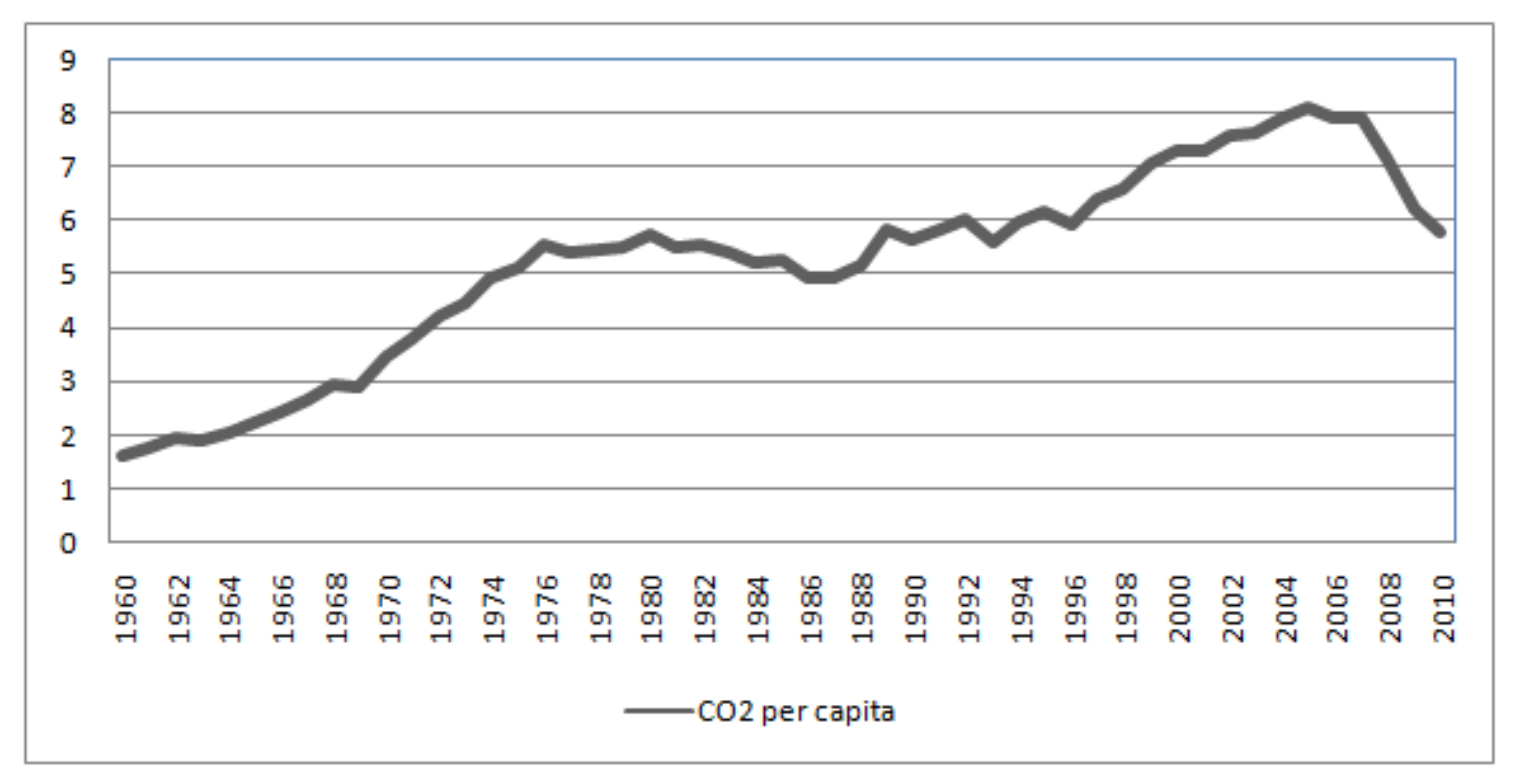

Figura 5. Emisiones de CO2 per cápita en España (1960-2010). (Banco Mundial)

Según Moreno (2005), los efectos del cambio climático en el turismo de nieve son una realidad, las evidencias del impacto del cambio climático en el turismo de invierno son de doble naturaleza. Por una parte, las evidencias científicas, los diversos estudios, que demuestran el leve aumento de las temperaturas en la montaña, el menor grosor medio de nieve y la mayor irregularidad temporal y territorial de la innivación. En la montaña española las precipitaciones en forma de nieve tienden a reducirse y las temperaturas en invierno tienden a aumentar (particularmente en febrero y marzo).

No es de extrañar, por tanto, que el concepto de desarrollo sostenible esté tomando relevancia. En este respecto la Unión Mundial por la Naturaleza define el desarrollo sostenible como un proceso que permite que se produzca el desarrollo sin deteriorar y agotar los recursos que lo hacen posible. Este 
objetivo se logra, generalmente, gestionando los recursos de forma que se puedan ir renovando al mismo ritmo que van siendo empleados, o pasando de un recurso que se regenera lentamente a otro que tenga un ritmo más rápido de regeneración. De esta forma, los recursos podrán seguir manteniendo a las generaciones presentes y futuras (McIntyre, 1993).

Como indican acertadamente Saz y Carús (2008) alcanzar el desarrollo sostenible en el turismo de nieve es harto complicado, en la medida en que los intereses de los diferentes agentes económicos tienen visiones de corto plazo sujetas a los criterios de rentabilidad.

Hasta la fecha, diversos estudios han analizado con mayor o menor detalle la afectación del cambio climático en algunas de las principales regiones alpinas del mundo. La mayoría de estos estudios se han centrado en los Alpes (König \& Abegg, 1997; Breiling \& Charamza, 1999; Elsasser \& Bürki, 2002; Abegg, Agrawala, Crick \& De Montfalcon, 2007; Steiger, 2010; Steiger \& Abegg, 2013; Uhlmann, Goyette \& Beniston, 2009), Canadá (McBoyle \& Wall, 1987; Scott, McBoyle \& Mills, 2003; Scott, McBoyle \& Minogue, 2007) y los Estados Unidos (Dawson \& Scott, 2010; Dawson, Scott \& Havitz, 2013), aunque países como Suecia (Moen \& Fredman, 2007), Australia (Hennessy, Whetton, Smith, Bathols, Hutchinson \& Sharples, 2003; Bicknell \& Mc-Manus, 2006), Japón (Fukushima, Kureha, Ozaki, Fujimori \& Harasawa, 2003) o Nueva Zelanda (Hendrikx, Hreinsson, Clark \& Mullan, 2012) también han sido analizados. Sin embargo solo el estudio realizado por Pons et al. (2014) analiza los Pirineos abarcando la zonas de Andorra, España y Francia en donde evalúan la posible influencia del cambio climático en la evolución del turismo de nieve, y más específicamente el esquí alpino, obteniendo tres perfiles diferentes de estaciones de esquí dependiendo de su vulnerabilidad frente al cambio climático.

A pesar de las limitaciones y la heterogeneidad de metodologías utilizadas, la mayoría de estos estudios son congruentes en señalar que en el futuro, el cambio climático conllevará a un impacto negativo en la duración de la temporada de esquí, la reducción de zonas esquiables y una disminución del número de esquiadores tanto en estaciones de baja altitud como de baja latitud por ello el principal objetivo de este trabajo es analizar la rentabilidad y situación financiera de las principales estaciones de esquí alpino catalanas, con el fin de comprobar si éstas son sostenibles desde un punto de vista económico. 


\section{Metodología}

Para realizar el estudio de rentabilidad y situación financiera de las estaciones de esquí, se han obtenido los estados financieros de dos fuentes de información diferenciadas, ya que el estudio incluye estaciones de esquí alpino de titularidad tanto privada como pública.

Los datos correspondientes a los estados financieros de estaciones de esquí de propiedad privada y/o explotación privada, se han obtenido a través de la base de datos SABI (Sistema de Análisis de Balances Ibéricos) mediante una búsqueda realizada durante el mes de septiembre de 2016. Los criterios de búsqueda aplicados para seleccionar la muestra ha sido seleccionar las empresas que se corresponden con el Código Nacional de Actividad Económica (CNAE), 9311: "Gestión de instalaciones deportivas", de ámbito geográfico Catalán que estuvieran activas en la actualidad y con datos disponibles para los períodos 2011-2015. Concretamente de la base SABI se obtienen los estados financieros de las estaciones de Baqueira-Beret, Masella, BoíTaüll y Vallter 2000. Por otro lado, los estados financieros correspondientes a las estaciones de esquí de propiedad pública: La Molina, Vall de Núria y Espot Esquí-Port Ainé, se obtienen de los Informes anuales publicados por la empresa pública Ferrocarriles de la Generalitat de Cataluña (FGC).

Respecto a la titularidad de las empresas hay que comentar que la estación Vallter 2000, era de propiedad privada hasta el 30 de junio de 2012, pasando a partir de esa fecha, a ser mayoritariamente de propiedad pública, ya que la Generalitat de Cataluña a través de la empresa pública FGC adquirió el $60.24 \%$ de su capital. No obstante, la explotación de la estación sigue realizándola la empresa privada Vallter, S.A. Las estaciones de Spot Esquí-Port Ainé fueron integradas en el año 2011 por lo que comparten estados financieros y no puede realizarse el análisis por separado.

Por último se plantea la duda de si incluir o no la estación de Boí Taüll, ya que en la base SABI los últimos datos disponibles son del ejercicio 2012, año en el que la empresa se encuentra en situación concursal. Finalmente se opta por incluirla con el fin de conocer cuál era su situación económica y financiera en ese momento. En diciembre de 2014 la estación pasó a ser propiedad de la Generalitat de Cataluña mediante la adquisición, a través de Avançsa, del crédito de 7.3 millones que la empresa Promocions Turístiques de la Vall, del grupo madrileño Nozar, tenía con el Instituto Catalán de Finanzas. Tras un acuerdo, se decidió que la empresa madrileña continuaría con su gestión durante un período de cinco años más, mediante un contrato de arrendamiento con opción a compra tras ese período y bajo un plan de viabilidad establecido por la Generalitat de Cataluña con el fin de reflotar el complejo turístico. 
Se han excluido de la muestra las estaciones de esquí que se dedican al esquí nórdico o de fondo, por no considerarse actividades que puedan compararse al esquí alpino, tanto por el impacto económico como por el número de usuarios. También se ha excluido de la muestra la estación de Tavascán por su escasa capacidad (dispone únicamente de dos remontadores).

\section{Análisis económico financiero}

\subsection{Análisis de la rentabilidad}

Con el objetivo de conocer cuál es la situación económica de las estaciones de esquí alpino catalanas, se procede a realizar la aplicación de un conjunto de ratios económicos.

\subsubsection{Rentabilidad económica $(\mathrm{ROA})=$ BAIT $/$ Total Activo}

La rentabilidad económica o ROA (ReturnonAssets) es el BAIT (Beneficio antes de Gastos Financieros y Tributos) generado, en relación a la inversión realizada para obtenerlo. O dicho de otro modo, es el rendimiento del activo.

\begin{tabular}{|c|c|c|c|c|c|}
\hline & 2011 & 2012 & 2013 & 2014 & 2015 \\
\hline Baqueira-Beret & $5.74 \%$ & $7.21 \%$ & $7.58 \%$ & $6.80 \%$ & $6.71 \%$ \\
\hline Masella & $10.30 \%$ & $6.73 \%$ & $4.62 \%$ & $3.75 \%$ & \\
\hline Vallter 2000 & $-4.68 \%$ & $-8.91 \%$ & $-18.42 \%$ & $-16.07 \%$ & $-23.02 \%$ \\
\hline Boí Taüll & $-3.16 \%$ & $-26.29 \%$ & - & - & \\
\hline La Molina & $-5.29 \%$ & $-6.07 \%$ & $-6.15 \%$ & $-7.05 \%$ & $-7.38 \%$ \\
\hline Vall de Núria & $-5.37 \%$ & $-5.91 \%$ & $-5.11 \%$ & $-6.10 \%$ & $-4.32 \%$ \\
\hline Spot esquí-Port Ainé & $-7.38 \%$ & $-7.35 \%$ & $-7.45 \%$ & $-8.48 \%$ & $-5.02 \%$ \\
\hline
\end{tabular}

Tabla 4. Rentabilidad económica o del activo (ROA). (SABI y memorias anuales de FGC)

En la Tabla 4 se observa que las estaciones de Masella y Baqueira-Beret son las únicas que obtienen una rentabilidad económica positiva. Aunque Masella presenta una tendencia decreciente año tras año, pasando de una rentabilidad del 10.3\% en 2011 a tan sólo 3.75\% en 2014. Mientras que Baqueira-Beret presenta un comportamiento irregular pero más estable. 
La Figura 6 muestra como el resto de estaciones, obtienen una rentabilidad económica negativa en todos los períodos analizados, ya que el BAIT de todas ellas es negativo.

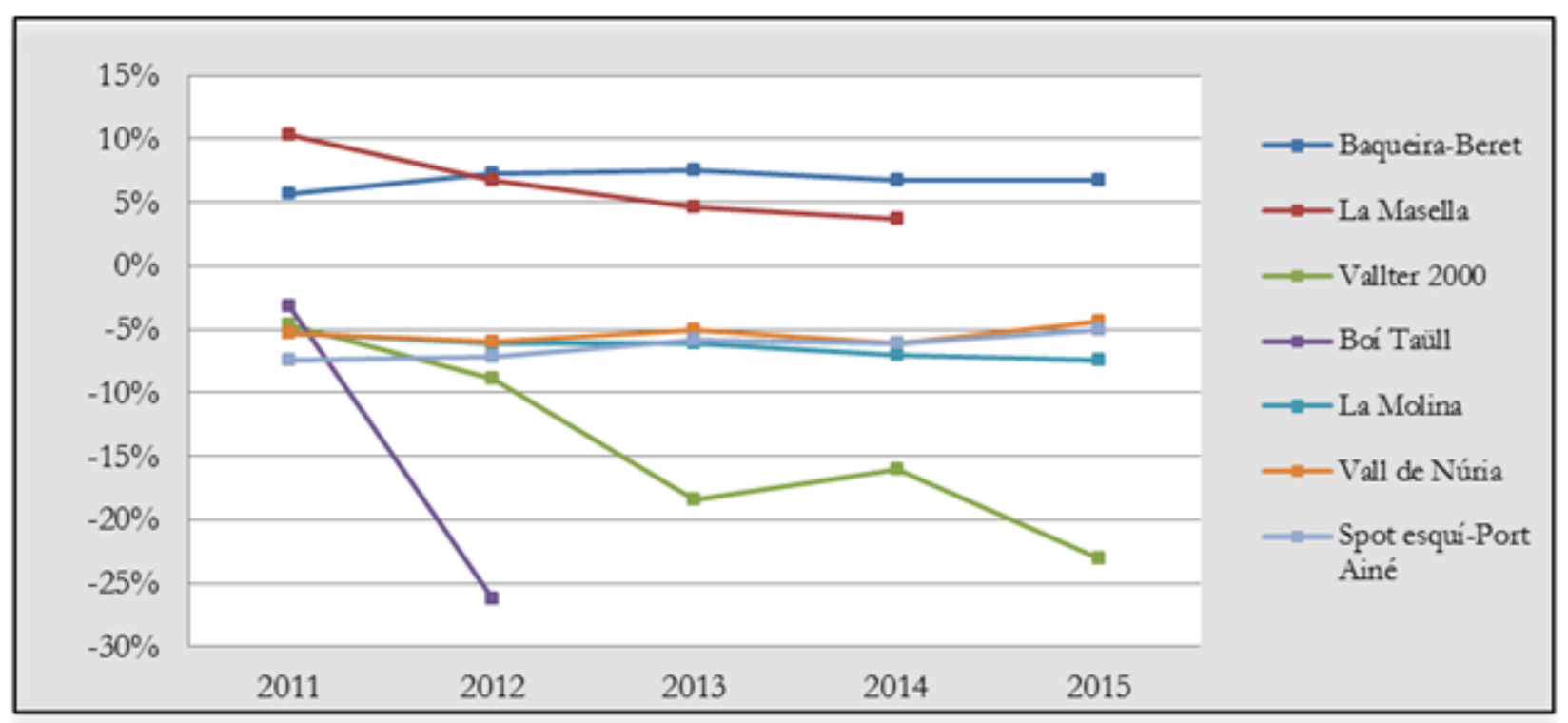

Figura 6. Rentabilidad económica (ROA). (SABI y memorias anuales de FGC)

Se sabe que la actividad de las estaciones de esquí alpino es una actividad acentuadamente intensiva en costes fijos (Massons, 2007), debido a que prácticamente todo su activo, es activo no corriente y los costes de estructura suelen ser muy elevados.

Del análisis de los estados financieros se observa cómo el coste de las amortizaciones es muy significativo, llegando en algunos casos a alcanzar un porcentaje sobre ventas de hasta el $99.27 \%$ en Vall de Núria y de un 81.57\% en Vallter 2000 para el año 2014, frente al 13.23\% de Masella y el 23.13\% de Baqueira-Beret. Los gastos de personal en porcentaje sobre ventas llegan hasta un 64\% en Vallter 2000 y a un $67 \%$ en Spot-Port Ainé en 2014, frente al 24.4\% de Masella y el 33.5\% de Baqueira-Beret. Por último, el coste derivado de Otros gastos de explotación, dónde se incluyen los consumos de energéticos, alcanzan un porcentaje sobre ventas del 69.16\% en Masella y un 61.8\% en Spot-Port Ainé en 2014, mientras que en Baqueira-Beret son del 30.33\% para ese mismo período. Estudios realizados por autores como Damm, Köberl y Prettenthaler (2014) o Steiger (2011) afirman que las estaciones de esquí en Austria, están en situaciones similares a las analizadas, el riesgo de afrontar un aumento sustancial de los costes totales de energía debido a los crecientes costes de electricidad esperados y por 
tanto su rentabilidad se vería afectada. Dorocki y Brzegowy (2014) analizan las estaciones de esquí polacas y llegan a las mismas conclusiones.

Con el fin de realizar un análisis más detallado sobre la rentabilidad económica (ROA) y siguiendo el modelo Dupont (González-Pascual, 2003), se estudian los dos factores que contribuyen en su obtención: el margen y la rotación del activo (Aramayo, Arimany, de Uribe-Salazar \& Sabata, 2013; Massons, 2007).

\subsubsection{Margen $=$ BAIT $/$ Ventas}

En la siguiente tabla se observa que las estaciones de Baqueira-Beret y Masella son las únicas que obtienen un margen positivo, ya que su BAIT también lo es. Aunque el comportamiento del margen en ambas estaciones ha sido distinto. Por una parte, se observa cómo Baqueira-Beret ha mejorado sus márgenes año tras año, mientras que Masella ha experimentado una evolución decreciente.

\begin{tabular}{|c|c|c|c|c|c|}
\hline & 2011 & 2012 & 2013 & 2014 & 2015 \\
\hline Baqueira-Beret & $11.87 \%$ & $14.43 \%$ & $16.33 \%$ & $16.14 \%$ & $15.37 \%$ \\
\hline Masella & $19.33 \%$ & $14.34 \%$ & $9.12 \%$ & $7.20 \%$ & - \\
\hline Vallter 2000 & $-30.45 \%$ & $-51.76 \%$ & $-124.44 \%$ & $-111.92 \%$ & $-181.63 \%$ \\
\hline BoíTaüll & $-27.81 \%$ & $-304.18 \%$ & - & - & - \\
\hline La Molina & $-54.58 \%$ & $-70.81 \%$ & $-55.23 \%$ & $-65.94 \%$ & $-63.22 \%$ \\
\hline Vall de Núria & $-57.49 \%$ & $-72.49 \%$ & $-62.58 \%$ & $-75.27 \%$ & $-47.08 \%$ \\
\hline Spot esquí-Port Ainé & $-258.24 \%$ & $-30.88 \%$ & $-48.83 \%$ & $-61.42 \%$ & $-33.26 \%$ \\
\hline
\end{tabular}

Tabla 5. Margen. (SABI y memorias anuales de FGC)

Esto puede explicarse porque Baqueira-Beret del año 2011 al 2014 ha disminuido sus costes fijos. Concretamente, los costes de personal, otros gastos de explotación (dónde se incluyen los gastos energéticos) y el gasto de amortización se han reducido de media, en un 9.27\%. En cambio, Masella en los períodos comprendidos entre 2011 y 2014, ha aumentado esos mismos gastos en una media del $8.55 \%$.

Para el resto de estaciones se observa márgenes negativos en todos los casos, ya que su BAIT también lo es. Llama especialmente la atención que los costes de personal, otros gastos de explotación y las amortizaciones del inmovilizado, son, en términos relativos respecto a las ventas, mucho mayores que los que soporta la estación de Baqueira-Beret. No obstante, en la Tabla 5 se observa que en 2015 las 
estaciones de La Molina, Vall de Núria y Spot esquí-Port Ainé mejoran su margen, que aunque continúa mostrando signo negativo, no es tan acentuado como en los períodos anteriores. Esto se debe a que todas ellas han reducido sus costes fijos sobre ventas. Concretamente, la Molina los reduce en un $5.28 \%$, Vall de Núria en un $9.58 \%$ y Spot esquí- Port Ainé en un $15.49 \%$ respecto al mismo período del año anterior. Por otro lado, Vallter 2000 en 2015 aumenta sus costes fijos sobre ventas en un 48.43\% respecto a 2014.

\subsubsection{Rotación del activo $=$ Ventas $/$ Total Activo}

En cuanto a la rotación del activo, es decir el número de veces que la inversión o activo se transforma en cifra de ventas en un año, es muy reducida en todas las estaciones. Esto es lógico debido a que la estaciones de esquí alpino necesitan de una gran inversión para llevar a cabo su actividad. Por tanto se podría decir que en este tipo de actividades, con elevada infraestructura, la rotación contribuye en menor medida que el margen en la obtención de la rentabilidad económica.

\begin{tabular}{|l|r|r|r|r|r|r|}
\cline { 2 - 7 } \multicolumn{1}{c|}{} & $\mathbf{2 0 1 1}$ & $\mathbf{2 0 1 2}$ & $\mathbf{2 0 1 3}$ & $\mathbf{2 0 1 4}$ & $\mathbf{2 0 1 5}$ \\
\hline Baqueira-Beret & 0.48 & 0.50 & 0.46 & 0.42 & 0.44 \\
\hline Masella & 0.53 & 0.47 & 0.51 & 0.52 & - \\
\hline Vallter 2000 & 0.15 & 0.17 & 0.15 & 0.14 & - \\
\hline BoíTaüll & 0.12 & 0.09 & -13 & - \\
\hline La Molina & 0.10 & 0.09 & 0.11 & 0.11 & 0.12 \\
\hline Vall de Núria & 0.09 & 0.08 & 0.08 & 0.08 & 0.1 \\
\hline Spot esquí-Port Ainé & 0.03 & 0.24 & 0.15 & 0.14 & 0.15 \\
\hline
\end{tabular}

Tabla 6. Rotación del activo. (SABI y memorias anuales de FGC)

No obstante, si se observa la Tabla 7 se ve como las estaciones de Masella y Baqueira-Beret son las que tienen mayor rotación, siendo estas estaciones las que presentan una mayor cifra de ventas. Contrariamente, las estaciones de Boí Taüll y Vall de Núria son las que muestran una rotación menor, muy cercana a cero.

\begin{tabular}{|l|r|r|r|r|r|}
\cline { 2 - 6 } \multicolumn{1}{c|}{} & \multicolumn{1}{c|}{$\mathbf{2 0 1 1}$} & $\mathbf{2 0 1 2}$ & $\mathbf{2 0 1 3}$ & \multicolumn{1}{c|}{$\mathbf{2 0 1 4}$} & \multicolumn{1}{c|}{$\mathbf{2 0 1 5}$} \\
\hline Baqueira-Beret & $24,712.49$ & $25,361.19$ & $24,509.72$ & $24,463.84$ & $26,208.09$ \\
\hline Masella & $8,063.98$ & $7,702.72$ & $6,636.73$ & $7,693.03$ & - \\
\hline Vallter 2000 & 814.99 & 814.99 & 734.65 & 671.04 & 619.27 \\
\hline BoíTaüll & $8,046.54$ & $4,842.30$ & - & - & - \\
\hline La Molina & $5,336.50$ & $4,226.30$ & $5,189.90$ & $4,686.20$ & $4,837.00$ \\
\hline Vall de Núria & $5,668.30$ & $4,839.80$ & $4,766.10$ & $4,786.30$ & $5,215.00$ \\
\hline Spot esquí-Port Ainé & 450.70 & $3,740.00$ & $2,562.90$ & $2,316.90$ & $2,742.00$ \\
\hline
\end{tabular}

Tabla 7. Importe neto de la cifra de negocios (miles de euros). (SABI y memorias anuales de FGC) 
En la Figura 7 se puede observar el importe neto de la cifra de negocios de cada una de las estaciones, siendo las estaciones de Baqueira-Beret y Masella las que obtienen mayor cifra de ventas. Se constata que en el año 2015 todas las estaciones analizadas, excepto Vallter 2000, aumentan su cifra neta de negocios. Por otro lado, Baqueira-Beret destaca como la estación líder y a gran distancia del resto.

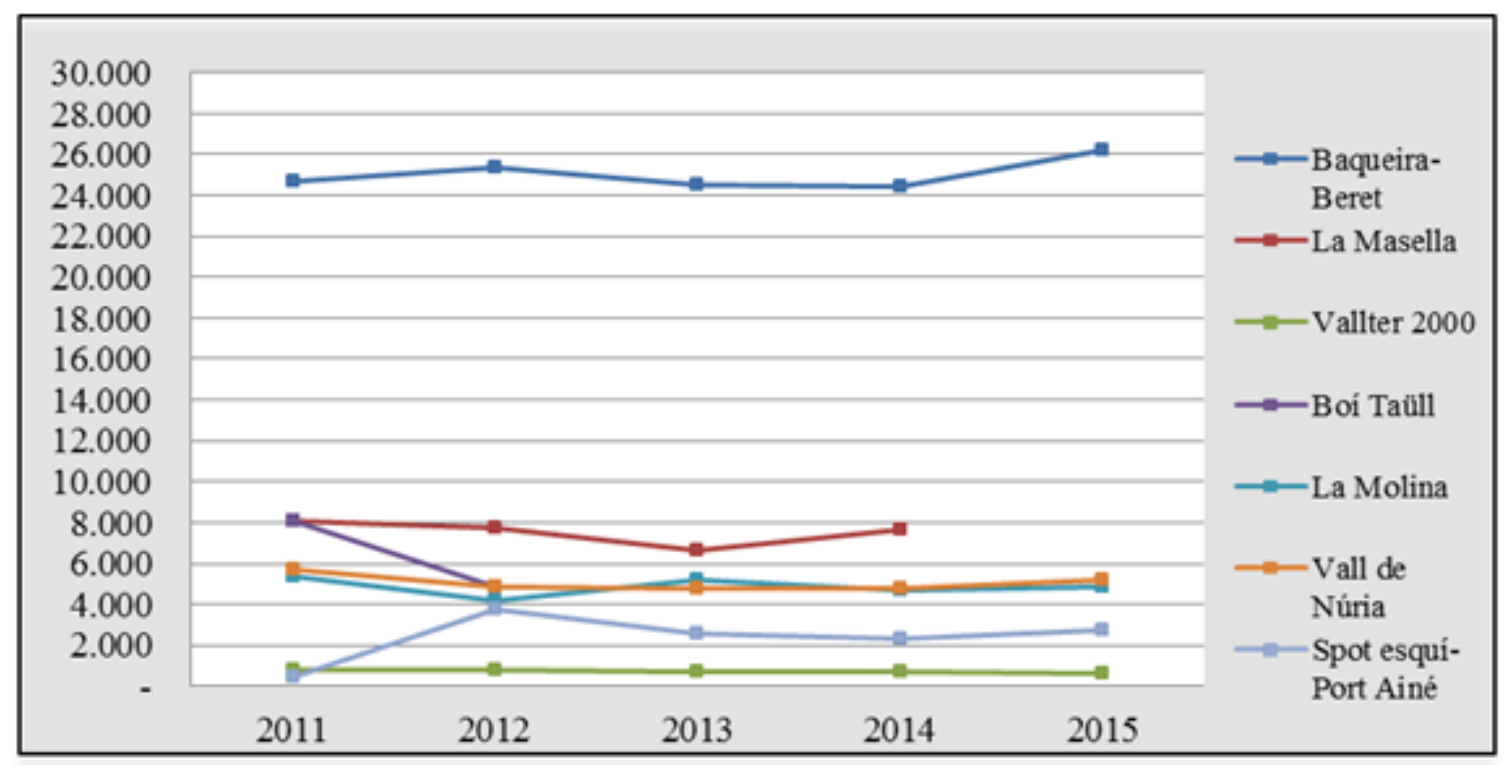

Figura 7. Importe neto de la cifra de negocios (miles de euros). (SABI y memorias anuales de FGC)

\subsubsection{Rendimiento de Explotación = EBIT /Activo funcional}

Asimismo, se ha calculado el rendimiento de explotación, que relaciona el Beneficio Neto de Explotación (EBIT) obtenido en relación al activo funcional o productivo utilizado para obtenerlo. El EBIT se diferencia del BAIT en que el primero no tiene en cuenta los ingresos financieros que se obtienen de los activos extrafuncionales o no productivos, mientras que el segundo sí.

\begin{tabular}{|c|c|c|c|c|c|}
\hline & 2011 & 2012 & 2013 & 2014 & 2015 \\
\hline Baqueira-Beret & $6.82 \%$ & $9.06 \%$ & $10.90 \%$ & $10.28 \%$ & $9.14 \%$ \\
\hline Masella & $12.43 \%$ & $9.76 \%$ & $4.58 \%$ & $4.89 \%$ & - \\
\hline Vallter 2000 & $-4.77 \%$ & $-9.05 \%$ & $-20.64 \%$ & $-16.57 \%$ & $-23.26 \%$ \\
\hline BoíTaüll & $-3.25 \%$ & $-26.90 \%$ & - & - & - \\
\hline La Molina & $-5.44 \%$ & $-5.92 \%$ & $-5.32 \%$ & $-6.08 \%$ & $-6.84 \%$ \\
\hline Vall de Núria & $-4.75 \%$ & $-6.13 \%$ & $-5.36 \%$ & $-6.40 \%$ & $-4.58 \%$ \\
\hline Spot esquí-Port Ainé & $-3.54 \%$ & $-7.41 \%$ & $-7.19 \%$ & $-6.77 \%$ & $-5.35 \%$ \\
\hline
\end{tabular}

Tabla 8. Rendimiento de Explotación. (SABI y memorias anuales de FGC) 
Las estaciones de Baqueira-Beret y Masella son las únicas que obtienen rendimiento de explotación positivo en todos los períodos analizados. Como se observa en la Tabla 8 no se aprecian variaciones muy significativas respecto a los resultados obtenidos para la rentabilidad económica. Esto se explica porque la rentabilidad de las estaciones de esquí se obtiene prácticamente en su totalidad de los activos productivos o funcionales. Para el resto de estaciones de esquí alpino, la situación no es nada alentadora, ya que el rendimiento de explotación es negativo todos los años (Figura 5). Esto indica que las estaciones tienen un problema económico, ya que ninguna de ellas está cubriendo el punto muerto. Es decir, ninguna alcanza un nivel de ventas suficiente como para cubrir los costes fijos y variables derivados de su actividad.

Especialmente destacan las estaciones de Boí Taüll que en 2012 obtiene un rendimiento de explotación de -26.90\% y de Vallter 2000 con un rendimiento de explotación de -20.64\% en 2013, -16.57\% en 2014 y $-23.26 \%$ en 2015 .

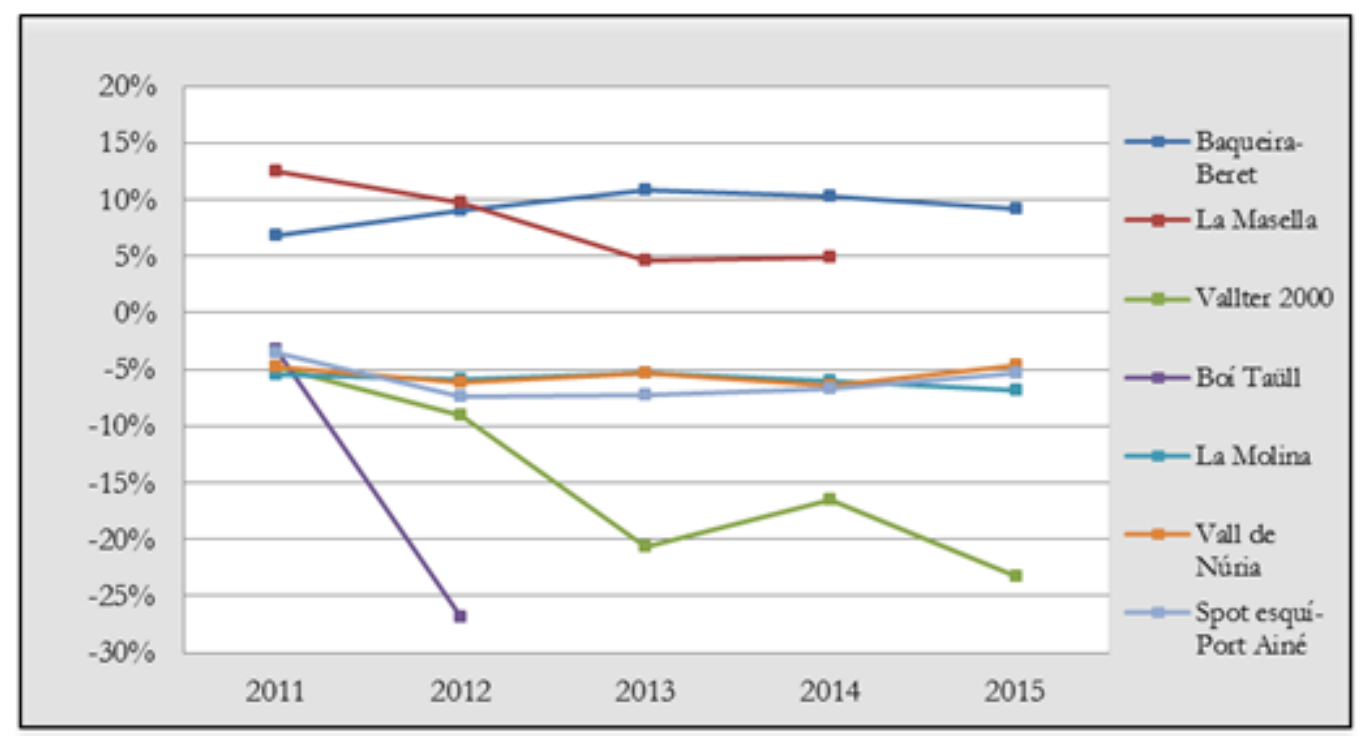

Figura 8. Rendimiento de Explotación. (SABI y memorias anuales de FGC)

Por último, se ha calculado el EBITDA en términos absolutos (véase Tabla 9) o Resultado Bruto de Explotación, con el propósito de analizar si al considerar únicamente el resultado de explotación antes de Intereses, Tributos, Amortizaciones y Depreciaciones, alguna de las estaciones que no alcanza el punto muerto, ahora sí lo hace. Como es sabido, el gasto de las amortizaciones es en cierta manera 
“flexible" ya que la propia ley permite aplicar métodos de amortización económica más o menos acelerados, lo cual influiría también en el resultado de explotación, haciéndolo menor o mayor en función del ritmo aplicado.

En la Tabla 9 se observa cómo los resultados no mejoran significativamente en ninguna de las estaciones con rendimiento de explotación negativo. No obstante, se observa que la estación de Vall de Núria mejora su EBITDA en 2015 en un 41.62\% respecto al año 2014, aunque éste continúa mostrando signo negativo. Esto es debido a la reducción de otros gastos de explotación sobre ventas en un $-1.43 \%$ y también a los gastos de personal sobre ventas en un $5.32 \%$ respecto al mismo período del año anterior. Por tanto, estos resultados ponen de manifiesto que, aunque no se considere el gasto de las amortizaciones y depreciaciones, la mayoría de estaciones siguen mostrando un resultado bruto de explotación (EBITDA) negativo, lo cual aún empeora más si cabe su problema económico.

\begin{tabular}{|l|r|r|r|r|r|}
\cline { 2 - 6 } \multicolumn{1}{c|}{} & \multicolumn{1}{c|}{$\mathbf{2 0 1 1}$} & \multicolumn{1}{c|}{$\mathbf{2 0 1 2}$} & $\mathbf{2 0 1 3}$ & \multicolumn{1}{c|}{$\mathbf{2 0 1 4}$} & \multicolumn{1}{c|}{$\mathbf{2 0 1 5}$} \\
\hline Baqueira-Beret & $8,170.27$ & $10,345.92$ & $9,805.87$ & $8,898.74$ & $9,628.85$ \\
\hline Masella & $2,877.10$ & $2,391.49$ & $1,365.34$ & $1,348.94$ & - \\
\hline Vallter 2000 & 190.41 & -115.67 & -259.11 & -238.96 & -481.41 \\
\hline BoíTaüll & 371.80 & $-6,988.91$ & - & - & - \\
\hline La Molina & 96.60 & -9.90 & 98.70 & -166.10 & -486.00 \\
\hline Vall de Núria & -222.70 & -505.30 & -598.00 & -772.50 & -451.00 \\
\hline Spot esquí-Port Ainé & -548.10 & $-1,138.70$ & $-1,195.80$ & $-1,374.50$ & $-1,361.00$ \\
\hline
\end{tabular}

Tabla 9. EBITDA (miles de euros). (SABI y memorias anuales de FGC)

\subsubsection{Rentabilidad de los accionistas $(\mathrm{ROE})=$ Beneficio Neto $/$ Fondos Propios}

La rentabilidad de los accionistas o ROE (ReturnonEquity) relaciona el Beneficio Neto obtenido, en relación a los fondos propios aportados por los propietarios.

\begin{tabular}{|l|r|r|r|r|r|}
\cline { 2 - 7 } \multicolumn{1}{c|}{} & \multicolumn{1}{c|}{$\mathbf{2 0 1 1}$} & $\mathbf{2 0 1 2}$ & $\mathbf{2 0 1 3}$ & \multicolumn{1}{c|}{$\mathbf{2 0 1 4}$} & \multicolumn{1}{c|}{$\mathbf{2 0 1 5}$} \\
\hline Baqueira-Beret & $5.34 \%$ & $6.12 \%$ & $6.64 \%$ & $5.94 \%$ & $6.00 \%$ \\
\hline Vassella & $11.81 \%$ & $7.74 \%$ & $3.57 \%$ & $3.54 \%$ & - \\
\hline BoíTaüll 2000 & $-75.78 \%$ & - & $-46.25 \%$ & $-59.89 \%$ & $-43.81 \%$ \\
\hline La Molina & $-17.82 \%$ & $-646.89 \%$ & - & - & - \\
\hline Vall de Núria & $-8.02 \%$ & $-8.88 \%$ & $-9.23 \%$ & $-10.96 \%$ & $-11.88 \%$ \\
\hline Spot esquí-Port Ainé & $-6.67 \%$ & $-7.70 \%$ & $-7.10 \%$ & $-10.34 \%$ & $-7.26 \%$ \\
\hline
\end{tabular}

Tabla 10. Rentabilidad de los accionistas (ROE). (SABI y memorias anuales de FGC) 
En la Tabla 10 se observa como las estaciones de Baqueira-Beret y Masella son las únicas que muestran un resultado de la ROE positiva, ya que son las únicas estaciones con beneficios. Para el resto de estaciones analizadas, la ROE es negativa debido a que todas ellas tienen pérdidas. Además en el caso de la estación Spot Esquí-Port Ainé no es posible realizar el cálculo ya que además de tener pérdidas, también tienen fondos propios negativos, lo cual hace que el resultado no tenga sentido. Lo mismo ocurre con Vallter 2000 en el año 2012, dónde el ratio no puede calcularse.

$\mathrm{Al}$ analizar la evolución de la ROE para las estaciones con beneficios, se observa que Baqueira-Beret tiene un comportamiento relativamente estable, con valores de la ROE que oscilan entre el 5.34\% y el 6.64\%. Mientras que en Masella se reduce año tras año debido a que sus beneficios también lo hacen, principalmente a la caída de los ingresos financieros y el aumento simultáneo de sus costes fijos de explotación.

También se puede constatar que Baqueira-Beret obtiene una ROE (Tabla 10) inferior a la ROA (Tabla 4) en todos los períodos analizados. A pesar de que Baqueira-Beret tiene un apalancamiento financiero positivo todos los años, éste apenas contribuye a la mejora de la rentabilidad que obtienen los propietarios, ya que su endeudamiento financiero o con coste es muy reducido (entre un 0.05 y un 0.11) y por tanto, la rentabilidad que podría obtener de la deuda financiera, ejerce un efecto casi nulo en la ROE.

En el caso de Masella, la situación es bastante similar, ya que aunque muestra un apalancamiento financiero positivo en casi todos los períodos, su endeudamiento financiero o con coste también es muy reducido (entre el 0.11 y el 0.85 ) y esto no ayuda a mejorar la rentabilidad de los fondos propios.

En general y a excepción de Boí Taüll y Vallter 2000 en 2012, en todos los años estudiados, se constata que las estaciones de esquí tienen un nivel de endeudamiento financiero muy reducido (por debajo de la unidad) lo cual no favorece a mejorar la rentabilidad de los accionistas o ROE. 


\subsection{Análisis de la estructura financiera}

Con el objetivo de conocer cuál es la situación financiera de las estaciones de esquí alpino catalanas, se procede a realizar la aplicación de un conjunto de ratios de estructura.

\subsubsection{Ratio de Endeudamiento = Pasivo exigible $/$ Fondos Propios}

El ratio de endeudamiento relaciona la proporción de deudas respecto a los fondos propios. Interesa que el valor de esta ratio sea lo más reducido posible, ya que de esta forma, la empresa disfrutará de mayor autonomía financiera (Massons, 2007). En la Tabla 11, se puede observar como el nivel de endeudamiento de la mayoría de empresas es muy reducido, situándose en valores muy por debajo de la unidad. Exceptuando las estaciones de Boí Taüll, Vallter 2000 y Spot-PortAiné.

\begin{tabular}{|l|r|r|r|r|r|r|}
\cline { 2 - 7 } \multicolumn{1}{c|}{} & \multicolumn{2}{c|}{$\mathbf{2 0 1 1}$} & $\mathbf{2 0 1 2}$ & $\mathbf{2 0 1 3}$ & $\mathbf{2 0 1 4}$ & $\mathbf{2 0 1 5}$ \\
\hline Baqueira-Beret & 0.19 & 0.16 & 0.18 & 0.18 & 0.19 \\
\hline Masella & 0.78 & 0.78 & 0.30 & 0.42 & - \\
\hline Vallter 2000 & 10.83 & -65 & 21.05 & 1.37 & -56 & - \\
\hline BoíTaüll & 2.65 & - & 0.09 & 0.05 & 0.07 \\
\hline La Molina & 0.11 & 0.06 & 0.17 & 0.18 & 0.19 \\
\hline Vall de Núria & 0.07 & 0.12 & - & - & - \\
\hline Spot esquí-Port Ainé & - & - & - & & \\
\hline
\end{tabular}

Tabla 11. Ratio de Endeudamiento. (SABI y memorias anuales de FGC)

Boí Taüll en 2012, refleja una situación de endeudamiento muy elevado, ya que su pasivo es 21.05 veces mayor a sus fondos propios. En 2012 la empresa se encuentra en situación de concurso de acreedores, después de varios años arrastrando importantes problemas económicos. En ese momento, la empresa está totalmente descapitalizada, ya que posee unos fondos propios de tan sólo el $4.52 \%$ del total patrimonio neto y pasivo, debido a la acumulación de pérdidas.

Como se observa en la Tabla 11, no se ha podido calcular el ratio de endeudamiento de Vallter 2000 en el año 2012 ni tampoco de Spot esquí-Port Ainé en ninguno de los ejercicios analizados, ya que éstas tienen en su balance fondos propios negativos, debido a la acumulación de pérdidas. Precisamente es en Julio de 2012, cuando la Generalitat de Cataluña sale al rescate de la estación Vallter 2000, adquiriendo el $60.24 \%$ de su capital. No obstante, en la Tabla 11, se observa como en el ejercicio 2015, la estación Vallter 2000 mejora significativamente su ratio de endeudamiento pasando de un 2.56 en 2014 a un 0.87 
en 2015, esto se debe a que en 2014 se realizó una ampliación de capital que aumentó sus fondos propios en un $100.03 \%$ y que se refleja en los estados financieros de 2015 . A su vez, para ese mismo período la estación disminuyó su pasivo corriente y no corriente en un $31.74 \%$ y $37.77 \%$ respectivamente.

Asimismo, Spot Esquí-Port Ainé muestra fondos propios negativos en todos los ejercicios, lo cual indica que la estación se encuentra en una situación de quiebra "técnica" salvada por las subvenciones que recibe de la Administración Pública, que hacen que su patrimonio neto se mantenga con signo positivo.

\subsubsection{Ratio de Liquidez o Fondo de Maniobra = Activo corriente/Pasivo corriente}

Esta ratio indica la capacidad que tiene una empresa para liquidar sus deudas de corto plazo, comparando las inversiones que deben transformarse en líquido en un período inferior o igual al año, con las deudas que se deben liquidarse en un año. Cuando el valor de esta ratio se sitúa por encima de 1, indica que el fondo de maniobra es positivo y como más se acerque a 2, más lejos estará la empresa del concurso de acreedores.

\begin{tabular}{|l|r|r|r|r|r|r|}
\cline { 2 - 7 } \multicolumn{1}{c|}{} & \multicolumn{2}{|c|}{$\mathbf{2 0 1 1}$} & $\mathbf{2 0 1 2}$ & $\mathbf{2 0 1 3}$ & $\mathbf{2 0 1 4}$ & $\mathbf{2 0 1 5}$ \\
\hline Baqueira-Beret & 1.12 & 1.40 & 2.10 & 2.40 & 1.7 \\
\hline Masella & 2.83 & 2.79 & 5.09 & 2.31 & - \\
\hline Boítraüll & 0.05 & 0.01 & 0.23 & -14 & - \\
\hline La Molina & 1.80 & 0.40 & - & 0.73 & 1.49 & 1.18 \\
\hline Vall de Núria & 1.50 & 1.24 & 0.68 & 0.67 & 0.81 \\
\hline Spot esquí-Port Ainé & 1.53 & 0.49 & 0.25 & 0.14 & 0.20 \\
\hline
\end{tabular}

Tabla 12. Ratio Liquidez o Fondo de Maniobra. (SABI y memorias anuales de FGC)

En la Tabla 12 se observa como las estaciones de Masella, Baqueira-Beret y la Molina (exceptuando 2013 para ésta última) obtienen resultados que superan la unidad, incluso algunos años muy por encima, como es el caso de Masella en 2013. Estos resultados indican que su fondo de maniobra es positivo y en principio, no deberían tener problemas de liquidez a corto plazo.

No ocurre lo mismo con el resto de estaciones y especialmente con Vallter 2000 dónde se obtienen resultados muy cercanos a cero todos los años, poniendo de manifiesto que su fondo de maniobra es 
negativo y que su capacidad para afrontar sus obligaciones de pago a corto plazo está en peligro. En el ejercicio 2014 su activo corriente es tan sólo de un 9.74\% frente al 71.07\% de su pasivo corriente, aunque de éste el 62.5\% corresponde a deudas a corto plazo con empresas del grupo y asociadas, lo que hace pensar que al ser una empresa participada mayoritariamente por la Generalitat a través de la empresa pública FCG, el riesgo de impago lo asume la propia entidad pública.

En la Figura 9 se observa como la estación de Masella es la que presenta mayor fondo de maniobra, seguida de Baqueira-Beret y La Molina. Mientras que Vallter 2000 y Spot Esquí-Port Ainé son las que lo tienen más bajo, mostrando resultados muy por debajo de la unidad.

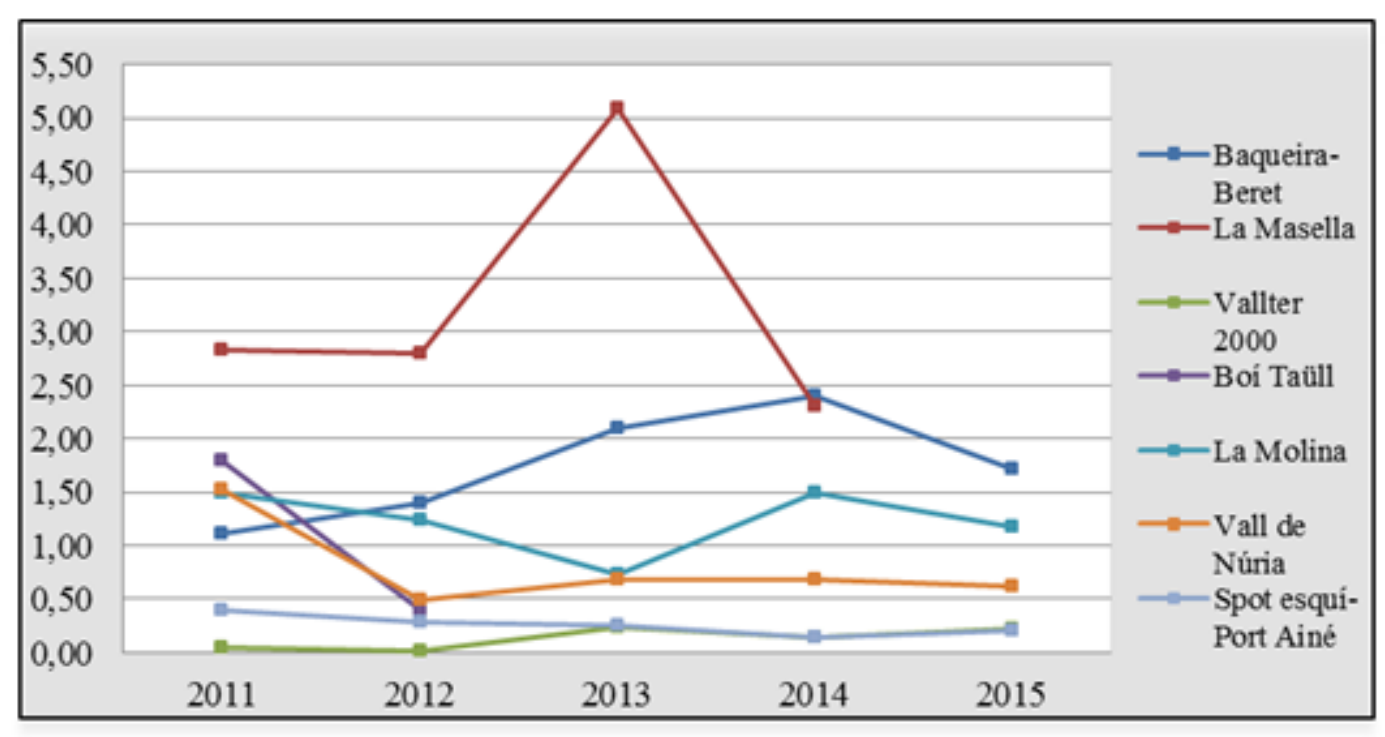

Figura 9. Ratio de Liquidez o Fondo de Maniobra. (SABI y memorias anuales de FGC)

\subsubsection{Ratio de Garantía $=$ Total activo $/$ Total pasivo}

En la Tabla 13 se ve como el valor del ratio de garantía o "distancia de la quiebra" da resultados muy elevados para las estaciones de La Molina, Vall de Núria, Baqueira-Beret y Masella. Esto ocurre porque son estaciones con elevada capitalización y muy poco endeudamiento tanto global como financiero. Esta ratio relaciona la capacidad total de la empresa (en caso de liquidación) para afrontar el pago de sus deudas con el total activos de los que dispone. Por tanto, como mayor sea el resultado, más garantía dará la empresa a los acreedores de que cobraran sus deudas. 


\begin{tabular}{|l|r|r|r|r|r|r|}
\cline { 2 - 7 } \multicolumn{1}{c|}{} & \multicolumn{2}{|c|}{$\mathbf{2 0 1 1}$} & $\mathbf{2 0 1 2}$ & $\mathbf{2 0 1 3}$ & $\mathbf{2 0 1 4}$ & $\mathbf{2 0 1 5}$ \\
\hline Baqueira-Beret & 6.28 & 7.25 & 6.65 & 6.65 & 6.34 \\
\hline Masella & 2.33 & 2.33 & 4.41 & 3.44 & - \\
\hline Ballter 2000 & 1.09 & 0.98 & 1.74 & -40 & 2.15 \\
\hline La Molina & 1.38 & 1.05 & - & - & - \\
\hline Vall de Núria & 13.82 & 25.99 & 16.24 & 28.63 & 27.27 \\
\hline Spot esquí-Port Ainé & 17.65 & 10.90 & 8.22 & 9.44 & 9.03 \\
\hline
\end{tabular}

Tabla 13. Ratio de Garantía. (SABI y memorias anuales de FGC)

Se puede observar como Vallter 2000 en el año 2012 presenta un valor por debajo de la unidad, lo que significa que en ese ejercicio, las deudas de la empresa eran mayores que su activo real. Es decir, la empresa se encuentra en una situación de quiebra "técnica" ya que su patrimonio neto es negativo debido a la acumulación de pérdidas.

La estación Boí-Taüll en el año 2012 también se acerca peligrosamente a la unidad, que se explica porque ese año la empresa se encuentra en situación concursal. Spot Esquí-Port Ainé también muestra valores por debajo de la unidad en los años 2011 y 2012, por la acumulación de pérdidas. No obstante el valor de su Patrimonio Neto en balance se muestra con signo positivo (excepto en el año 2012), gracias a las subvenciones que recibe.

\section{Resultados}

Los resultados del estudio ponen de manifiesto que las estaciones de Baqueira-Beret y Masella son las únicas que obtienen una rentabilidad económica o ROA positiva, mientras que el resto de estaciones obtienen una rentabilidad económica negativa en todos los períodos analizados. Estos resultados son similares a los obtenidos en el trabajo realizado por Massons (2007) en el que se analizaba la rentabilidad económica de las estaciones de esquí en el período comprendido entre 1993-1998. En este caso, si bien es cierto que no daban rendimientos negativos estas estaciones eran las que presentaban una mayor rentabilidad económica.

En referencia al análisis de los factores que contribuyen a la obtención de la rentabilidad económica; esto es el margen y la rotación de activos, los resultados muestran que la rotación del activo es reducida en todas las estaciones, siendo las de Masella y Baqueira-Beret las que tienen mayor rotación, coincidiendo con que también son, las que tienen mayor cifra de ventas. Así mismo las únicas estaciones que obtienen un margen positivo son Baqueira-Beret y Masella. 
El rendimiento de explotación también es negativo en todas las estaciones, excepto en la de BaqueiraBeret y Masella, por tanto el estudio revela que la mayoría de estaciones tienen un gran problema económico, ya a que ninguna de ellas está cubriendo el punto muerto. Es decir, ninguna alcanza un nivel de ventas suficiente como para cubrir los costes fijos y variables derivados de su actividad. Del análisis de las cuentas de resultados, se observa que el coste de las amortizaciones en este tipo de actividad, es muy significativo, así como los gastos de personal y también el coste derivado de "Otros gastos de explotación", dónde se incluyen los consumos de energéticos. Los estados financieros de las diferentes estaciones analizadas, revelan que éstas, realizan una actividad con elevado apalancamiento económico, ya que ante una variación porcentual de sus ventas, la disminución (o aumento) del BAIT es varias veces superior, por el efecto "palanca" que ejercen los costes fijos. Son empresas a las que alcanzar el punto muerto les es más difícil, por ese mismo motivo: ante una disminución de las ventas o ventas insuficientes, sus gastos de explotación prácticamente no se reducen, porque la mayoría de ellos son fijos. Lo cual supone un gran riesgo económico.

Por último y respecto a la situación financiera, se observa que el nivel de endeudamiento global y financiero de las estaciones de esquí es muy reducido (por debajo de la unidad) lo cual no favorece a mejorar la rentabilidad que obtienen los accionistas o ROE para el caso de Baqueira-Beret y Masella, que son las únicas estaciones con beneficios. Por tanto, el problema fundamental de las estaciones de esquí alpino catalanas, es un problema económico más que financiero.

\section{Conclusiones}

En este trabajo se ha analizado la sostenibilidad económica de las estaciones de esquí del Pirineo Catalán así como los factores externos que pueden influir en sus resultados. En él hemos podido constatar que la crisis económica que ha atravesado nuestro país en los últimos años, unida a que la práctica del esquí no se considera una actividad de turismo de masas, ha llevado a un estancamiento de la cifra de negocios en la mayoría de estaciones Catalanas.

A esta situación se une al problema de la falta de nieve, producida por el cambio climático y la necesidad de las estaciones de producir nieve artificial, aumentando de manera considerable los costes y disminuyendo la eficiencia energética e hídrica. Estudios realizados en estaciones de Austria, Polonia, los Alpes, Estados Unidos, Suecia, Australia, Japón o Nueva Zelanda llegan a las mismas conclusiones. Haciendo que la actividad de estas empresas sea escasamente sostenible económicamente tal como se 
refleja en el actual análisis realizado en Cataluña, las únicas estaciones rentables a nivel económico, son las estaciones privadas Baqueira-Beret y Masella.

El resto de estaciones del Pirineo Catalán han sido rescatadas o adquiridas por la Generalitat, ya que en vez de generar beneficios se han convertido actualmente en centros de costes que promueven la rentabilidad de otros negocios como; restauración, comercio, hoteles, empresas de formación y de actividades complementarias, generando puestos de trabajo y actuando como motores económicos, pero que sólo tienen sentido y razón de ser, si las temporadas invernales son largas para la práctica de deportes de invierno.

Por otra parte, los municipios con estaciones de esquí han incrementado su población. Sin embargo han disminuido sus activos referentes al sector primario, creando cierta dependencia del sector servicios que actualmente está en declive debido a la reducción de la temporada de esquí. El enfrentamiento entre turismo y actividades primarias supone un gran problema para el desarrollo sostenible que debe basarse en la complementariedad de ambas. Si las actividades turísticas perjudican al sector primario a no muy largo plazo se perderán los recursos naturales y conllevará a la degradación del paisaje.

En definitiva, de los resultados observados y con las limitaciones derivadas de la muestra utilizada, se deduce que, en la mayoría de los casos, el mantenimiento de las estaciones de esquí alpino catalanas no es sostenible, si se mira desde un punto de vista estrictamente económico e incierto desde un punto de vista ambiental. El estudio realizado revela que, para la mayoría de ellas, la ayuda de la Administración ha sido y es, absolutamente necesaria para su sostenimiento.

Por lo que se deduce, la Administración, las mantiene por las rentas que genera su existencia. Es decir, en las externalidades positivas que genera en el territorio donde se ubican.

Se recomienda que se implementen estrategias para conseguir diversificar la oferta como por ejemplo la creación de parques de aventura lúdicos de invierno, instalaciones para la realización de deportes específicos de interior y de exterior, con el objetivo de mantener los negocios satélites de las actuales estaciones de esquí y paliar las pérdidas generadas por la caída de los ingresos de las actividades relacionadas con el turismo de nieve que en su día fueron alternativas económicas para las zonas rurales que se quedaban sin población. Por tanto hay que buscar nuevas fórmulas que compatibilicen el turismo con el desarrollo económico del territorio manteniendo la biodiversidad y el paisaje, requisitos imprescindibles para la sostenibilidad de áreas donde la naturaleza es su principal recurso. 
Una de las limitaciones del trabajo es que las conclusiones se obtienen a partir de un período de análisis de cinco años (2011-2015) por lo que el alcance de las mismas, no pueden generalizarse en todos los casos.

\section{Referencias}

Abegg, B., Agrawala, S., Crick, F., \& De Montfalcon, A. (2007). Climate change impacts and adaptation in winter tourism. In S. Agrawala (Ed.), Climate change in the European Alps: Adapting winter tourism and natural bazards management (pp. 25-58). París: Organization for Economic Cooperation and Development.

Aramayo, A., Arimany, N., de Uribe-Salazar, C., \& Sabata, A. (2013). Economic and financial analysis sector catalan meat. Intangible Capital, 9(2), 437-458. https:/doi.org/10.3926/ic.459

Arnáez, J., Lasanta, T., \& Pascual-Bellido, N. (2014). La contribución de una pequeña estación de esquí al desarrollo de su entorno: El caso de Valdezcaray (La Rioja). Cuadernos de Turismo, 33, 151-172.

Beniston, M. (2003). Climatic change in mountain regions: A review of possible impacts. Climatic Change, 59, 5-31. https:/doi.org/10.1023/A:1024458411589

Bicknell., S., \& McManus, P. (2006).The canary in the coalmine: Australian ski resorts and their response to climate change. Geographical Research, 44, 386-400. https:/doi.org/10.1111/j.17455871.2006.00409.x

Breiling, M., \& Charamza, P. (1999).The impact of global warming on winter tourism and skiing: A regionalized model for Austrian snow conditions. Regional Environmental Change, 1(1), 4-14. https:/doi.org/10.1007/s101130050003

Clarimont, S. (2008). Turismo de invierno y cambio climático: La producción de nieve artificial en los Pirineos, ¿un uso sostenible del agua?. $6^{\circ}$ Congreso Ibérico sobre gestión y planificación del agua (pp. 1-11). Vitoria.

Damm, A., Köberl, J., \& Prettenthaler, F. (2014). Does artificial snow production pay under future climate conditions?-A case study for a vulnerable ski area in Austria. Tourism Management, 43, 8-21. https:/doi.org/10.1016/j.tourman.2014.01.009

Dawson, J., \& Scott, D. (2010). Systems analysis of climate change vulnerability for the US Northeast ski sector. Tourism Planning and Development, 7(3), 219-235. https:/doi.org/10.1080/1479053X.2010.502383 
Dawson, J., Scott, D., \& Havitz, M. (2013). Skier demand and behavioural adaptation to climate change in the US Northeast. Leisure/Loisir, 37(2), 127-143. https:/doi.org/10.1080/14927713.2013.805037

Dorocki, S., \& Brzegowy, P. (2014). Ski and spa tourism as local development strategy-the case of KrynicaZdrój (Poland). Annales Universitatis Paedagogicae Cracoviensis Studia Geographica, 5, 88-116.

El Kenawy, A., López-Moreno, J.I., \& Vicente-Serrano, S.M. (2012). Trend and variability of temperature in northeastern Spain (1920-2006): Linkage to atmospheric circulation. Atmospheric Research, 106, 159-180. https:/doi.org/10.1016/j.atmosres.2011.12.006

Elsasser, H., \& Bürki, R. (2002). Climate change as a threat to tourism in the Alps. Climate Research, 20, 253-257. https:/doi.org/10.3354/cr020253

Esteban-Talaya, A. (1995). Los nuevos desarrollos turísticos en España y su efecto en la recuperación y/o promoción económica. Papers de Turisme, 17, 33-44.

Falk, M. (2009). Are multi-resort ski conglomerates more efficient?. Managerial and Decision Economics, 30(8), 529-538. https:/doi.org/10.1002/mde.1470

Falk, M. (2010). A dynamic panel data analysis of snow depth and winter tourism. Tourism Management, 31(6), 912-924. https:/doi.org/10.1016/j.tourman.2009.11.010

Fukushima, T., Kureha, M., Ozaki, N., Fujimori, Y., \& Harasawa, H. (2003). Influences of air temperature change on leisure industries: Case study on ski activities. Mitigation and Strategies for Climate Change, 7, 173-189. https:/doi.org/10.1023/A:1022803405470

García-Abad A. (2012). Estaciones de esquí, turismo y entorno rural de montaña: Claves para una regulación de las estaciones de esquí ante el cambio climático. Acciones e Investigaciones Sociales, 31(julio), 91-135.

Gerbaux, F., \& Marcelpoil, E. (2006). Gouvernance des stations de montagne en France: Les spécificités du partenariat public-privé. La Revue de Géograpbie Alpine, 94(1), 9-19. https:/doi.org/10.3406/rga.2006.2380

Gómez-Martín, M.B. (2003). Duración y características de la estación climático-turística estival en Cataluña. Estudios Geográficos, 64(253), 623-653. https:/doi.org/10.3989/egeogr.2003.i253.201

González Pascual, J. (2003). Análisis de la Empresa a través de su información económico financiera. Fundamentos teóricos y aplicaciones. Madrid: Editorial Pirámide. 
Hendrikx, J., Hreinsson, E.Ö., Clark, M.P., \& Mullan, A.B. (2012). The potential impact of climate change on seasonal snow in New Zealand: Part I an analysis using 12 GCMs. Theoretical and Applied Climatology, 110(4), 607-618. https:/doi.org/10.1007/s00704-012-0711-1

Hennessy, K., Whetton, P., Smith, I., Bathols, J., Hutchinson, M., \& Sharples, J. (2008). Climate change on snow conditions in mainland Australia and adaptation at ski resorts through snowmaking. Climate Research, 35, 255-270. https:/doi.org/10.3354/cr00706

Instituto Estadístico de Cataluña (IDESCAT) (2016). Población: Comarcas, ámbitos y provincias. Disponible online en: http://www.idescat.cat/. (Fecha última consulta: 10 de octubre, 2016).

IPCC (Intergovernmental Panel on Climate Change) (2014). Climate Change 2014-Impacts, Adaptation and Vulnerability: Regional Aspects. Cambridge University Press. Disponible online en: https://www.ipcc.ch/pdf/assessment-report/ar5/wg2/ar5 wgII spm es.pdf. (Fecha última consulta: 3 de febrero, 2016).

Jiménez, S., \& Prats, L. (2006). El turismo en Cataluña: evolución histórica y retos de futuro. PASOS. Revista de Turismo y de Patrimonio Cultural, 4(2), 153-174.

Keller, F., Kienast, F., \& Beniston, M. (2000). Evidence of the response of vegetation to Environmental Change at High Elevation Sites in the Swiss Alps. Regional Environmental Change, 1(2), 70-77. https:/doi.org/10.1007/PL00011535

König, U., \& Abegg, B. (1997). Impacts of climate change on tourism in the Swiss Alps. Journal of Sustainable Tourism, 5(1), 46-58. https:/doi.org/10.1080/09669589708667275

Lasanta, T. (2010). El turismo de nieve como estrategia de desarrollo en el Pirineo Aragonés. Cuadernos de Investigación Geográfica, 36(2), 145-163. https:/doi.org/10.18172/cig.1242

Lasanta, T., Laguna, M., \& Vicente-Serrano, S.M. (2007). Do tourism-based ski resorts contribute to the homogeneous development of the Mediterranean mountains? A case study in the Central Spanish Pyrenees. Tourism Management, 28(5), 1.326-1.339. https:/doi.org/10.1016/j.tourman.2007.01.003

López-Palomeque, F. (1996). Turismo de invierno y estaciones de esquí en el Pirineo Catalán. Investigaciones Geográficas, 15, 19-39. https:/doi.org/10.14198/INGEO1996.15.04

López-Palomeque, F. (2004). La gestión pública del turismo en Cataluña: Organización y política turística de la administración autonómica. Investigaciones Geográficas, 34, 5-28. 
Martín, B.G. (2008). El turismo de nieve en España. El caso de Baqueira Beret (Cataluña). En: $A$ propósito del turismo: La construcción social del espacio turístico (pp. 292-302). Barcelona: Editorial UOC.

Martín, B.G., López-Palomeque, F., \& Vide, F.J. (2002). Aptitud climática y turismo. Variaciones geográficas y cronológicas de la potencialidad climático-turística del verano en Cataluña. Ería, 59, 333-345.

Massons, J. (2007). La rendibilitat de les estacions catalanes d'esqui alpí. Tesis doctoral. Escuela Superior de Administración y Dirección de Empresas. ESADE. Barcelona. 383.

McBoyle, G., \& Wall, G. (1987). The impact of Co2 induced warming on downhill skiing in the Laurentians. Cabiers de Géographie du Québec, 31(82), 39-50. https:/doi.org/10.7202/021843ar

McIntyre, G. (1993). Sustainable tourism development, guide for local planners. World Tourism Organization (WTO), 166.

Moen, J., \& Fredman, P. (2007). Effects of Climate Change on Alpine Skiing in Sweden. Journal of Sustainable Tourism, 15(4), 418-437. https:/doi.org/10.2167/jost624.0

Moreno, J.M. (Coord.) (2005). Evaluación preliminar de los impactos en España por efecto del cambio climático. Ministerio de Medio Ambiente. Disponible online en: http://www.magrama.gob.es (Fecha última consulta: 3 de febrero, 2016)

Pons, M., López-Moreno, J.I., Esteban, P., Macià, S., Gavaldà, J., García, C. et al. (2014). Influencia del cambio climático en el turismo de nieve del Pirineo. Experiencia del proyecto de investigación NIVOPYR. Pirineos, 169, e006. https:/doi.org/10.3989/Pirineos.2014.169006

Romagosa, F., Priestley, G.K., \& Llurdés, J.C. (2011). El turismo en el marco de una estrategia de planificación sostenible general en Cataluña. Boletín de la asociación de geógrafos españoles, 57, 267-294.

Saz, M.I., \& Carús L. (2008). La sostenibilidad del turismo recreativo de alta montaña. Cuadernos de Desarrollo Rural, 5(60), 11-36.

Schumpeter, J.A. (1927). La explicación de los Ciclos Económicos. Barcelona; Oikos-Tau.

Scott, D., McBoyle, G., Mills, B. (2003). Climate change and the skiing industry in southern Ontario (Canada): Exploring the importance of snowmaking as a technical adaptation. Climate Research, 23, 171-181. https:/doi.org/10.3354/cr023171

Scott, D., McBoyle, G., \& Minogue, A. (2007). Climate change and Quebec's ski industry. Global Environmental Change, 17(2), 181-190. https:/doi.org/10.1016/j.gloenvcha.2006.05.004 
Steiger, R. (2010). The impact of climate change on ski season length and snowmaking requirements in Tyrol, Austria. Climate Research, 43(3), 251-262. https:/doi.org/10.3354/cr00941

Steiger, R. (2011). The impact of snow scarcity on ski tourism: An analysis of the record warm season 2006/2007 in Tyrol (Austria). Tourism Review, 66(3), 4-13. https:/doi.org/10.1108/16605371111175285

Steiger, R., \& Abegg, B. (2013). The sensitivity of Austrian Ski areas to climate change. Tourism, Planning and Development, 10(4), 480-493. https:/doi.org/10.1080/21568316.2013.804431

Uhlmann, B., Goyette, S., \& Beniston, M. (2009). Sensitivity analysis of snow patterns in Swiss ski resorts to shifts in temperature, precipitation and humidity under condition of climate change. International Journal of Climatology, 29(8), 1048-1055. https:/doi.org/10.1002/joc.1786

WTO (World Tourism Organization) (2008). Climate change and Tourism. Responding to Global Challenges. Madrid. (pp. 269). Disponible online en: http://sdt.unwto.org/sites/all/files/docpdf/climate2008.pdf. (Fecha última consulta: 3 de febrero, 2016).

Intangible Capital, 2016 (www.intangiblecapital.org) 